Article

\title{
Seasonal Variation in Diurnal Photosynthesis and Chlorophyll Fluorescence of Four Genotypes of Cassava (Manihot esculenta Crantz) under Irrigation Conditions in a Tropical Savanna Climate
}

\author{
Supranee Santanoo ${ }^{1}$, Kochaphan Vongcharoen ${ }^{2}$, Poramate Banterng ${ }^{3}$ (D), Nimitr Vorasoot $^{3}$, \\ Sanun Jogloy ${ }^{3}$, Sittiruk Roytrakul ${ }^{4}$ and Piyada Theerakulpisut ${ }^{1,2, *}$ \\ 1 Department of Biology, Faculty of Science, Khon Kaen University, Khon Kaen 40002, Thailand; \\ supranee4705@hotmail.com \\ 2 Salt-Tolerant Rice Research Group, Department of Biology Faculty of Science Khon Kaen University, \\ Khon Kaen 40002, Thailand; kocha_9@hotmail.com \\ 3 Department of Agronomy, Faculty of Agriculture, Khon Kaen University, Khon Kaen 40002, Thailand; \\ pboram@kku.ac.th (P.B.); nvorasoot1@gmail.com (N.V.); sjogloy@gmail.com (S.J.) \\ 4 The National Center for Genetic Engineering and Biotechnology (BIOTEC), Phahonyothin Road Khlong \\ Nueng, Khlong Luang Pathum Thani 12120, Thailand; sittiruk@biotec.or.th \\ * Correspondence: piythe@kku.ac.th; Tel.: +66-43-202-531; Fax: +66-43-202-530
}

Received: 22 February 2019; Accepted: 18 April 2019; Published: 23 April 2019

\begin{abstract}
Photosynthesis performance during early vegetative growth is an important physiological trait determining yield of cassava, but limited information is currently available for the tropical savanna climate of Asia. Diurnal photosynthesis and chlorophyll fluorescence of the three-month-old plants of four commercial cassava genotypes (Rayong 9, RY9; Rayong 11, RY11; Kasetsart 50, KU50 and CMR38-125-77) grown under irrigation, were investigated in three seasons i.e., rainy, cool and hot. The mean daily net photosynthetic rate (Pn) across genotypes in the rainy season $\left(11.75 \mu \mathrm{molCO} / \mathrm{m}_{2}^{2} / \mathrm{s}\right)$ was significantly lower than that in the cool season $\left(14.60 \mu \mathrm{molCO} / 2 \mathrm{~m}^{2} / \mathrm{s}\right)$. Daily mean Pn in the hot season was $14.32 \mu \mathrm{molCO} / \mathrm{m}^{2} / \mathrm{s}$. In the rainy season, maximum photochemical quantum yield of PSII $\left(\mathrm{F}_{\mathrm{v}} / \mathrm{F}_{\mathrm{m}}\right)$ and effective quantum yield of PSII photochemistry $\left(\Phi_{\mathrm{PSII}}\right)$ were significantly higher than the other seasons, while electron transfer rate (ETR) and non-photochemical quenching (NPQ) were significantly lower. Genotypic variation was observed during the hot season in which RY11 had the highest and CMR38-125-77 the lowest mean daily Pn. The prominent mechanism to avoid damages from stress during afternoon in the hot season was to reduce leaf temperature by enhancing transpiration for RY11; to close stomata early for RY9, and to increase NPQ for CMR38-125-77.
\end{abstract}

Keywords: cassava genotypes; photosynthetic performance; photosystem II efficiency; climatic factors

\section{Introduction}

Cassava (Manihot esculenta Crantz) is one of the most significant crops for food security and the main food source for more than 0.8 billion people in Africa and Asia [1,2]. Cassava is also used to produce starch for industrial applications including paper, textile, food and beverages, plywood, glue, animal feed and ethanol [3]. Thailand is ranked as the world's largest exporter of cassava products, supplying around $67 \%$ of the global market with annual production of 31 million tons in 2016 [4]. The country exports cassava products in the form of dry chips, pellets, and native and modified starch [5]. Most of the cassava growing areas in Thailand are in the tropical savanna climate zone with a growing period, from planting to storage root harvesting, of eight to 12 months, which covers almost 
all three seasons in Thailand (hot season, March to May; rainy season, June to October, and cool season, November to February) [6].

Storage root yield of cassava varies considerably depending on cultivars, climate, growing conditions, crop management and planting time $[7,8]$. While the highest recorded experimental yield potential was 80 to $90 \mathrm{t} \mathrm{ha}^{-1}$ under near optimal edaphic-climatic conditions $[9,10]$, the average yield in Thailand was recorded at $18.83-24.15 \mathrm{t} \mathrm{ha}^{-1}$ [11]. This yield gap might be due to a lack of sufficient agro-advisory information about cassava genotypes suitable for planting in different seasons and appropriate management practices for the different growing seasons [12].

Crop yield is primarily determined by photosynthesis $[13,14]$ and net photosynthetic rate (Pn) of cassava leaves was reported to have a significant correlation with storage root yield across environments [15]. Related photosynthesis parameters such as internal $\mathrm{CO}_{2}$ concentration (Ci) was also significantly correlated with dry root yield of cassava [16]. In addition, changes in chlorophyll (Chl) fluorescence parameters under different environmental conditions can be used as a rapid and sensitive measure of plant health status, photosynthetic competence as well as effects of stress on plants $[17,18]$. Information on environmental effects in different seasons on photosynthetic performance of cassava genotypes is seriously lacking for the tropical savanna climate including Thailand. If seasonal variations in environmental conditions and responsive physiological parameters are precisely measured under specific field condition, it would help determine plant-based and/or environmental factors limiting photosynthetic performance as well as providing essential input data for effective crop growth and photosynthesis modeling [19]. This information will be useful for providing a set of guidelines in improving management of cultural practice of cassava growing in different seasons.

The effect of weather variations during different periods of growing seasons in Thailand on growth rate and final yield of cassava has been reported [12]. However, the physiological responses, particularly photosynthesis, of cassava genotypes in different growing seasons have not been evaluated in Thailand. Therefore, the objectives of this study were to investigate the effect of weather fluctuation in different seasons on diurnal photosynthesis and Chl fluorescence of the three-month-old plants of four commercially important cassava genotypes grown under irrigation to determine appropriate genotypes for planting in different seasons based on their growth and photosynthetic performance.

\section{Materials and Methods}

\subsection{Site Description}

The study site was at the experimental research station, Faculty of Agriculture, Khon Kaen University, Northeast Thailand $\left(16^{\circ} 28^{\prime} 29.7^{\prime \prime} \mathrm{N}, 102^{\circ} 48^{\prime} 37.3^{\prime \prime} \mathrm{E}\right.$, altitude $195 \mathrm{~m}$ above sea level). Soils were of the Yasothon soil series; fine loamy, siliceous, and Oxic Paleustult [20]. The climate is equatorial savanna with a dry winter [21]. The seasonal mean air temperatures for the Northeastern part of Thailand for 30 years (1981-2010) were $27.6,24.2$ and $28.6^{\circ} \mathrm{C}$, with the mean minimum of 24.4, 18.7 and $23.2{ }^{\circ} \mathrm{C}$, and mean maximum of $32.6,30.6$ and $35.2^{\circ} \mathrm{C}$ in rainy, cool and hot seasons, respectively. The average seasonal rainfalls, during 1981-2010, were $1103 \mathrm{~mm}, 76 \mathrm{~mm}$, and $224 \mathrm{~mm}$ in rainy, cool and hot seasons, respectively [22].

The experimental study was conducted during June 2015 to April 2016. Environmental characteristics including ambient PAR $\left(\mathrm{PAR}_{\mathrm{A}}\right)$, air temperature $\left(\mathrm{T}_{\text {air }}\right)$, air relative humidity $\left(\mathrm{RH}_{\text {air }}\right)$ and rainfall were recorded every five minutes by an automatic weather station (Watchdog 2000, Spectrum Technologies Inc., Lincoln, NE, USA). Air vapor pressure deficit $\left(\mathrm{VPD}_{\text {air }}\right)$ was calculated from air temperature, saturated vapor pressure and RH with the aid of Smithsonian Meteorological Tables prepared by Robert J. List, Smithsonian Institution Press, City of Washington [23] (Figure 1). The weather was under the influence of monsoon winds, specifically the southwest and northeast monsoon. In 2015-2016, the three seasons were divided as follows; rainy season or southwest monsoon season from June to October, cool season or northeast monsoon from November to February, and hot or 
pre-monsoon from March to May. The monthly mean environmental parameters in the experimental field are shown in Figure 1.

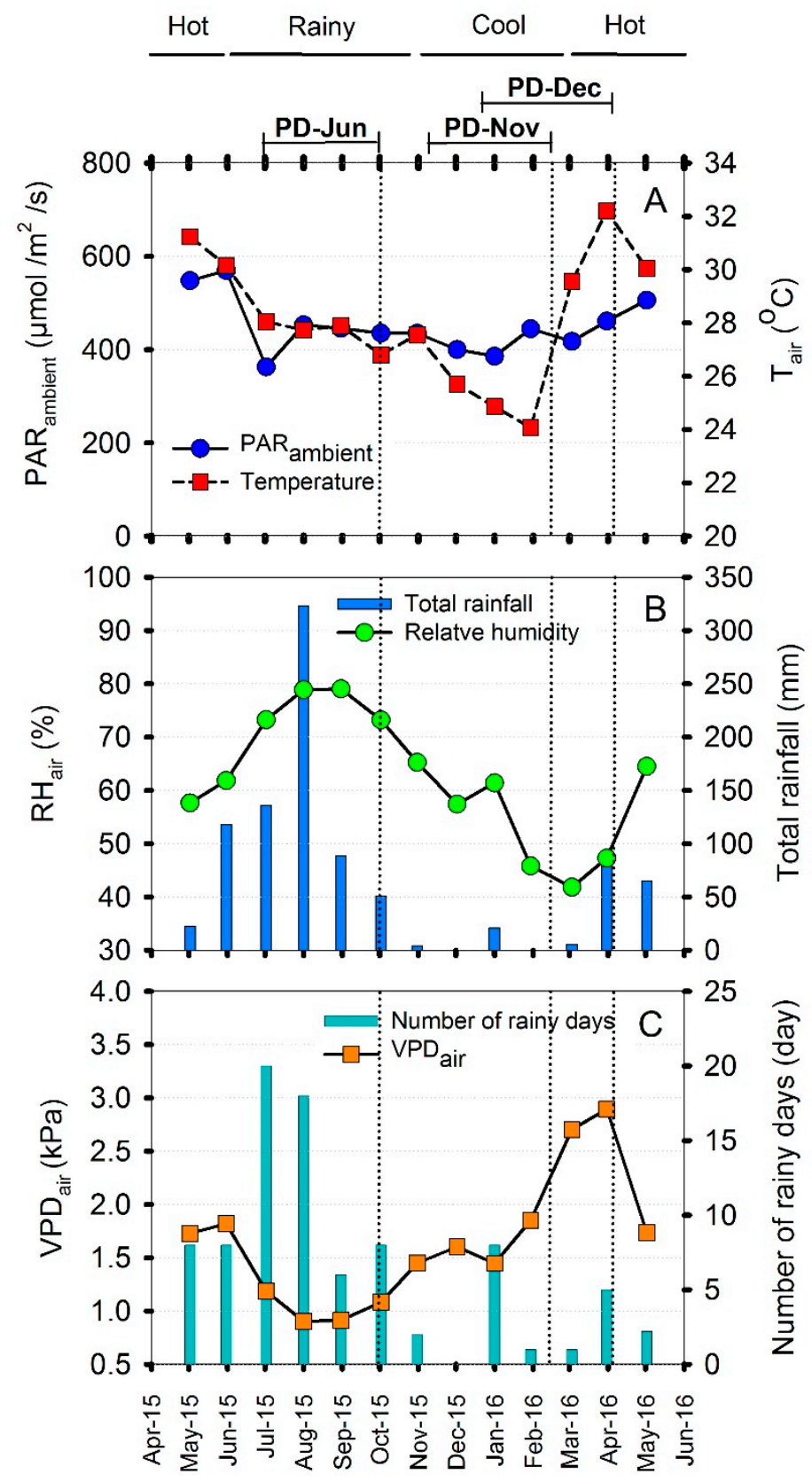

Figure 1. Monthly mean environmental parameters at the experimental site from May, 2015 to May, 2016. Daytime ambient photosynthetically active radiation $\left(\mathrm{PAR}_{\mathrm{A}}\right)$ and daytime air temperature $\left(\mathrm{T}_{\text {air }}\right)$ (A), relative humidity $\left(\mathrm{RH}_{\text {air }}\right)$ and total rainfall $(\mathbf{B})$, air vapor pressure deficit $\left(\mathrm{VPD}_{\text {air }}\right)$ and number of rainy days (C). Rainy season ranged from June to October, cool season from November to February, and hot from March to May. Dotted vertical lines indicate the timing of photosynthetic measurements made on three-month-old cassava plants in each season. Rainy season measurements were made in October 2015 on plants established on 30 June 2015 (PD-Jun); cool season measurements were made in February 2016 on plants established on 10 November 2015 (PD-Nov); and hot season measurements were made in April 2016 on plants established on 15 December 2015 (PD-Dec). 


\subsection{Plant Materials}

Four cassava genotypes were selected for physiological studies, all were improved cultivars or line with high starch content suitable for industrial uses. Two plant types are recognized i.e., one non-branching plant type (cv. Rayong 9, RY9), and one branching plant type (2 cvs. Rayong 11, RY11; and Kasetsart 50, KU50, and one line CMR-38-125-77). The four cassava genotypes were planted at three different planting dates (PD), namely, PD-Jun, PD-Nov and PD-Dec. PD-Jun plants were planted on 30 June 2015, PD-Nov on 10 November 2015 and PD-Dec on 15 December 2015. Stem cuttings (20 cm long) were planted with a plant spacing of $1 \mathrm{~m} \times 1 \mathrm{~m}$ in the plot size of $5 \mathrm{~m}$ wide and $7 \mathrm{~m}$ long. All cassava plants were fertilized and kept well-watered (soil matric potential was maintained between 0 to $-30 \mathrm{kPa})$ throughout the growing and measurement period. Nitrogen fertilizer $\left((\mathrm{NH})_{2} \mathrm{SO}_{4}\right)$ formula 21-0-0 was applied at one month after planting at the rate of $46.9 \mathrm{~kg} \mathrm{ha}^{-1}$ based on soil analysis and nutrient requirements for cassava $[23,24]$. At two months after planting the compound fertilizer $\mathrm{N}-\mathrm{P}_{2} \mathrm{O}_{5}-\mathrm{K}_{2} \mathrm{O}$ formula 15-0-18 was applied at a rate of $312.5 \mathrm{~kg} \mathrm{ha}^{-1}$ (Chia tai company limited, Phranakhonsiayutthaya, Krung Thep Maha Nakhon, Thailand). The plants were irrigated by a mini-overhead sprinkler irrigation system. Soil matric potential was continuously monitored by Tensiometer and water mark (Watchdog 1000, Spectrum Technologies Inc., Lincoln, NE, USA) at the soil depths of 20 and $40 \mathrm{~cm}$. Water applications were administered whenever soil matric potential reduced to $-30 \mathrm{kPa}$ at $20 \mathrm{~cm}$ depth. Rainy season measurements of growth and photosynthesis were performed on PD-Jun plants when they were three months old (in October 2015). Cool and hot season measurements were performed on three-month-old PD-Nov and PD-Dec plants in February and April 2016, respectively.

Plant growth rate was measured, on the planting date and after three months of planting, as the rate of increase in stem height $\left(\mathrm{cm} \mathrm{d}^{-1}\right)$, and rate of leaf production $\left(l e a f \mathrm{~d}^{-1}\right)$, on six randomly selected plants per genotype. Leaf area index (LAI) of the three-month-old plants was measured by gap fraction analysis using LI-191R line quantum sensor (Li-Cor Inc., Lincoln, NE, USA). Canopy structure was calculated according to the equation $\mathrm{LAI}=-(1 / \mathrm{k}) \ln \left(\mathrm{Q}_{\mathrm{b}} / \mathrm{Q}_{\mathrm{a}}\right)$ where $\mathrm{k}$ is assumed to be close to $0.5, \mathrm{Q}_{\mathrm{b}}$ is an average below-canopy PAR and $Q_{a}$ is an unobstructed PAR reading [25].

\subsection{Physiological Measurement}

Photosynthetic measurements were made on three-month-old plants on two days in each season. The three-month-old plants were selected for photosynthetic measurements because the most active vegetative growth occurs during the period three to six months after planting, and storage roots begin to be formed at this stage [26]. Rainy season measurements were made on 25 September and 2 October 2015 on plants established on 30 June 2015 (PD-Jun). Cool season measurements were made on 24 and 26 February 2016 on plants established on 10 November 2015 (PD-Nov). Hot season measurements were made on 3 and 5 April 2016 on plants established on 15 December 2015. On each day, diurnal variation in gas exchange and chlorophyll fluorescence were measured at $2 \mathrm{~h}$ intervals (eight time points) from 04:30 to 18:30 local time. For each time point, the measurements were performed on the central lobe of a young fully expanded leaf of two randomly selected plants of each genotype.

Gas exchange parameters were evaluated using a portable gas exchange system, infrared gas analyzer (LI-6400xt, Li-Cor Inc., Lincoln, NE, USA) equipped with the standard $2 \times 3 \mathrm{~cm}$ leaf chamber (6400-08 clear chamber bottom). Pn, Gs, Tr and $C_{i}$ were measured under ambient environmental conditions. The instantaneous light use efficiency (LUE) was calculated from Pn/absorbed photosynthetically active radiation. Water-use efficiency (WUE) was calculated as the ratio of $\mathrm{Pn} / \mathrm{Tr}$. Ratio between internal $\mathrm{CO}_{2}$ concentration and ambient $\mathrm{CO}_{2}(\mathrm{Ci} / \mathrm{Ca})$ was calculated to indicate non-stomatal limitation of photosynthesis.

Chl fluorescence subtly reflects the primary reactions of photosynthesis and is closely associated with photosystem II (PSII) photochemical efficiency. Chl fluorescence parameters were measured using Mini PAM-II Photosynthesis Yield Analyzer (Heinz Walz GmbH, Effeltrich, Germany). Minimal fluorescence yield of the dark-adapted state $\left(\mathrm{F}_{0}\right)$ was measured in complete darkness 
before sunrise (04:30). Maximal fluorescence of the dark-adapted state $\left(\mathrm{F}_{\mathrm{m}}\right)$ was obtained following a saturating pulse of $4000 \mu \mathrm{mol} / \mathrm{m}^{2} / \mathrm{s}$ lasting $0.8 \mathrm{~s}$. Maximal quantum yield of PSII photochemistry $\left(\mathrm{F}_{\mathrm{v}} / \mathrm{F}_{\mathrm{m}}\right)$ was calculated according to the equation $\mathrm{F}_{\mathrm{v}} / \mathrm{F}_{\mathrm{m}}=\left(\mathrm{F}_{\mathrm{m}}-\mathrm{F}_{0}\right) / \mathrm{F}_{\mathrm{m}}$. Steady state fluorescence in the light-adapted state $\left(\mathrm{F}^{\prime}\right)$ and the maximal fluorescence of the light-adapted state $\left(\mathrm{F}_{\mathrm{m}}{ }^{\prime}\right)$ were measured during the day between 06:30-16:30 (every two hours, 6 time points). Effective quantum yield of PSII photochemistry $\left(\Phi_{\mathrm{PSII}}\right)$ was calculated from the equation: $\Phi_{\mathrm{PSII}}=\left(\mathrm{F}_{\mathrm{m}}{ }^{\prime}-\mathrm{F}^{\prime}\right) / \mathrm{F}_{\mathrm{m}}{ }^{\prime}$ and nonphotochemical quenching $(\mathrm{NPQ})$ from: $\mathrm{NPQ}=\left(\mathrm{F}_{\mathrm{m}}-\mathrm{F}_{\mathrm{m}}{ }^{\prime}\right) / \mathrm{F}_{\mathrm{m}}{ }^{\prime}$. Electron transport rate (ETR) was calculated from the equation: $\mathrm{ETR}=\Phi_{\mathrm{PSII}} \times 0.84 \times 0.5 \times \operatorname{PAR}[27,28]$.

\subsection{Data and Statistical Analysis}

Comparisons among planting dates of mean ambient photosynthetically active radiation $\left(\mathrm{PAR}_{\mathrm{A}}\right)$, air temperature $\left(\mathrm{T}_{\text {air }}\right)$, air relative humidity $\left(\mathrm{RH}_{\text {air }}\right)$ and air vapor pressure deficit $\left(\mathrm{VPD}_{\text {air }}\right)$ were done by using paired $t$-test. Mean comparisons of growth, environmental, photosynthesis and chlorophyll fluorescence parameters were using one-way ANOVA for comparing means among genotypes within season and among seasons within each genotype, averages of multiple comparisons were determined by a Tukey's test under Sigmaplot version 11.0 software [29]. The correlation analysis between photosynthesis (Pn, Gs, Tr, $\Phi_{\mathrm{PSII}}, \mathrm{ETR}$ and NPQ) and environmental parameters including PAR $_{\mathrm{A}}$, $\mathrm{T}_{\text {leaf }}$, leaf-to-air vapor pressure deficit $\left(\mathrm{VPD}_{\mathrm{L}}\right)$ and relative humidity in the plant canopy $\left(\mathrm{RH}_{\mathrm{C}}\right)$ were analyzed in different seasons. Statistical significance was taken at $p<0.05$ and $p<0.01$ using MSTAT-C Version 1.42 software [30]. All statistical analyses followed the procedure described by Gomez and Gomez [31].

\section{Results}

\subsection{Environment and Plant Growth}

The environmental conditions under which the cassava plants of the three planting dates (PD-Jun, PD-Nov and PD-Dec) were growing were recorded from the date of planting until the plants were three-month-old as shown in Table S1 (see Supplementary). The values of mean daily PAR for the three planting dates were similar ranging from $412-429 \mu \mathrm{mol} / \mathrm{m}^{2} / \mathrm{s}$. Among the three planting dates, the PD-Jun plants experienced the highest daily minimum $\left(23^{\circ} \mathrm{C}\right)$ and highest daily mean temperature $\left(28^{\circ} \mathrm{C}\right)$. On the other hand, the PD-Nov and PD-Dec plants were exposed to the lowest daily minimum temperature of $19{ }^{\circ} \mathrm{C}$. The mean daily temperatures during growth of PD-Nov and PD-Dec plants were 25 and $26^{\circ} \mathrm{C}$, respectively. The highest mean daily RH was found for PD-Jun (77\%) while those recorded for PD-Nov and PD-Dec were at $57 \%$ and $52 \%$, respectively. The lowest daily minimum RH (27-31\%) was recorded in the cool season for the PD-Nov and PD-Dec plants. The PD-Jun plants which were growing in the rainy season were exposed to the lowest daily maximum $(2.55 \mathrm{kPa})$ and daily mean $(1.02 \mathrm{kPa}) \mathrm{VPD}_{\text {air }}$. The daily maximum VPD air values increased for PD-Nov $(3.58 \mathrm{kPa})$ and PD-Dec ( $4.03 \mathrm{kPa})$ plants which were growing during the cool and cool-to-hot seasons, respectively. The highest total rainfall was recorded for PD-Jun $(614.3 \mathrm{~mm})$ and the lowest $(25.6 \mathrm{~mm})$ for PD-Nov plants. In this experiment, irrigation was applied by a mini-overhead sprinkler system to maintain soil matric potential level at approximately $-30 \mathrm{kPa}$ or higher. The highest and lowest total irrigation were applied to PD-Dec $(41.6 \mathrm{~mm})$ and PD-Jun $(7.2 \mathrm{~mm})$ plants, respectively.

Cassava plant growth rates during the first three months after planting were measured as the rate of increase in plant height $\left(\mathrm{cm} \mathrm{d}^{-1}\right)$ and the rate of leaf production $\left(\right.$ leaf $\mathrm{d}^{-1}$ ) as shown in Figure 2 and Table S2. The rate of increase in plant height of plants established in June (PD-Jun plants) which were growing in the rainy season, averaged across four genotypes $\left(1.28 \mathrm{~cm} \mathrm{~d}^{-1}\right)$, was significantly higher $(p<0.001)$ than the rates for PD-Nov $\left(0.75 \mathrm{~cm} \mathrm{~d}^{-1}\right)$ and PD-Dec $\left(0.72 \mathrm{~cm} \mathrm{~d}^{-1}\right)$ plants which were planted and growing in the cool and cool-to-hot season, respectively (Figure 2A; Table S2). Rates of leaf production across four genotypes for all three planting dates were not significantly different. For PD-Jun plants, leaf production rates for CMR38-125-77 (0.85 leaf d $\left.\mathrm{d}^{-1}\right)$, RY11 (0.79 leaf $\left.\mathrm{d}^{-1}\right)$ and 
RY9 $\left(0.77\right.$ leaf $\left.\mathrm{d}^{-1}\right)$ were not significantly different while that of KU50 $\left(0.53\right.$ leaf $\left.\mathrm{d}^{-1}\right)$ was significantly lower than the others $(p<0.001)$. For plants established in December (PD-Dec), CMR38-125-77 showed significantly higher leaf production rate $(p=0.004)$ than the other genotypes (Table S2). It was noted that KU50 plants established in June and November had significantly lower $(p=0.010)$ leaf production rate than those in December (Table S2). Mean leaf area index (LAI) of the three-month-old plants across genotypes for PD-Jun plants (3.32) was significantly higher than $(p<0.001)$ those planted in the cool season (2.32 and 2.61 for PD-Nov and PD-Dec, respectively) (Figure 2C; Table S2). However, significant difference $(p<0.001)$ among cultivars was observed only in PD-Dec in which CMR38-125-77 had the highest mean LAI of 3.49 followed by RY11 (2.51), RY9 (2.45) and KU50 (2.00).

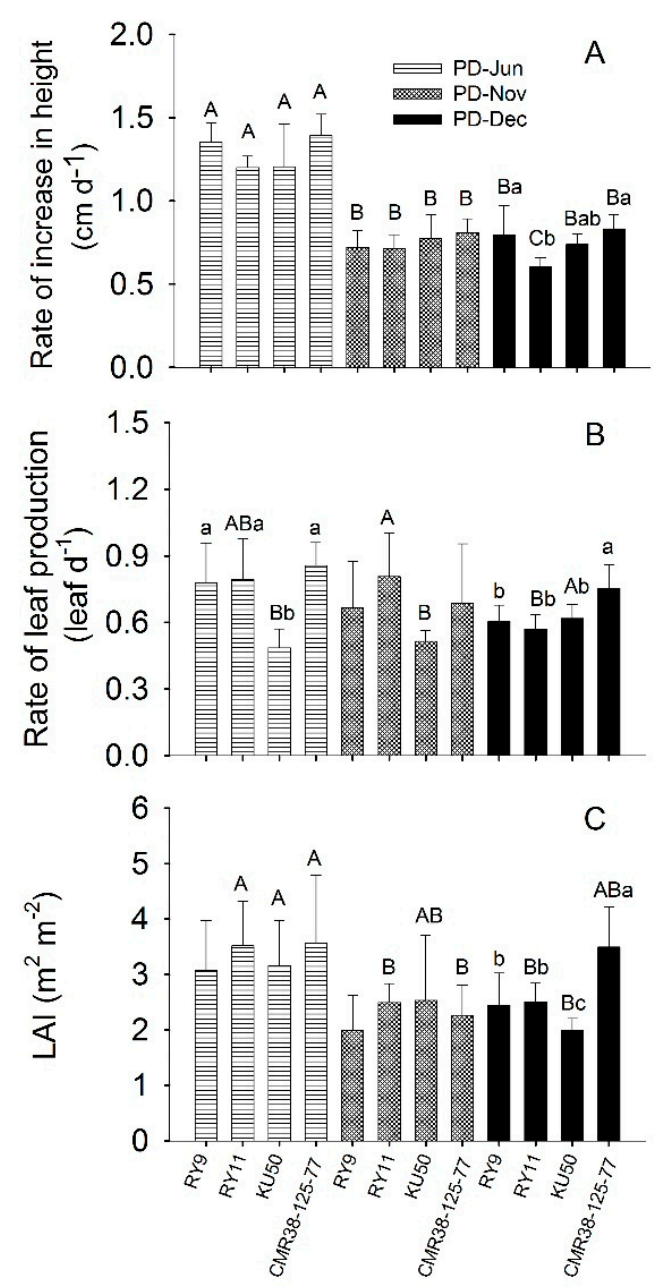

Figure 2. Rate of increase in plant height (A), rate of leaf production (B) and leaf area index (LAI) of three-month-old plants (C) of cassava genotypes RY9, RY11, KU50 and CMR38-125-77 planted in June 2015 (PD-Jun), November 2015 (PD-Nov) and December 2015 (PD-Dec). Means which are significantly different $(p<0.05)$ among genotypes for each planting date are denoted by different lower-case letters. For each genotype means which are significantly different among planting dates are denoted with capital letters. Data shows mean of six replicates \pm standard deviation (SD).

\subsection{Diurnal Variation in Environmental Conditions during Field Measurements of Photosynthesis}

Diurnal patterns of environments $\left(\mathrm{PAR}_{\mathrm{A}}, \mathrm{T}_{\mathrm{air}}, \mathrm{RH}_{\text {air }}\right.$ and $\left.\mathrm{VPD}_{\text {air }}\right)$ measured during 04:30-18:30 on two sunny days of photosynthesis measurements in each of the three seasons, compared with leaf/canopy parameters $\left(\mathrm{PAR}_{\text {leaf }}, \mathrm{T}_{\text {leaf }}, \mathrm{RH}_{\mathrm{C}}\right.$ and $\left.\mathrm{VPD}_{\mathrm{L}}\right)$ were depicted in Figure 3. For each season, the diurnal minima, maxima and means recorded during 06:30 to 16:30 across two days of investigation were summarized in Table 1. Diurnal variation in ambient PAR $\left(\mathrm{PAR}_{\mathrm{A}}\right)$ compared to incident PAR ( $\left.\mathrm{PAR}_{\text {leaf }}\right)$ 
were displayed in Figure 3A-F for measurements performed in rainy, cool and hot seasons, respectively. Similarly, the comparison between diurnal $\mathrm{T}_{\text {air }}$ and $\mathrm{T}_{\text {leaf }}$ were shown in Figure 3G-L. Diurnal $\mathrm{RH}_{\text {air }}$ compared to $\mathrm{RH}_{\mathrm{C}}$, and $\mathrm{VPD}_{\text {air }}$ compared to $\mathrm{VPD}_{\mathrm{L}}$ were demonstrated in Figure $3 \mathrm{M}-\mathrm{R}, \mathrm{S}-\mathrm{X}$, respectively. The daily minima, maxima and means of the environmental and leaf/canopy parameters recorded over the two days of photosynthesis measurements were shown in Table 1 . The $\mathrm{PAR}_{\text {leaf }}$ which were recorded on the top canopy leaves during gas exchange measurement were similar to $\mathrm{PAR}_{\mathrm{A}}$ over the canopy throughout the day except for the period during 12:00-15:00 when PAR leaf tended to be lower than $\mathrm{PAR}_{\mathrm{A}}$ (Figure 3A-F). There were seasonal differences $(p<0.001)$ in the mean daily $\mathrm{PAR}_{\text {leaf }}$ across genotypes, with the highest mean $\mathrm{PAR}_{\text {leaf }}$ in the hot season $\left(930 \mu \mathrm{mol} / \mathrm{m}^{2} / \mathrm{s}\right)$ followed by the $\operatorname{cool}\left(863 \mu \mathrm{mol} / \mathrm{m}^{2} / \mathrm{s}\right)$ and the rainy $\left(518 \mu \mathrm{mol} / \mathrm{m}^{2} / \mathrm{s}\right)$ seasons (Table 1$)$.

In the rainy season, $\mathrm{T}_{\text {leaf }}$ of all genotypes were higher than $\mathrm{T}_{\text {air }}$ on the first day (Figure $3 \mathrm{G}$ ) probably due to high $\mathrm{PAR}_{\mathrm{A}}$ (Figure $3 \mathrm{~A}$ ). However, on the second day (Figure $3 \mathrm{H}$ ) $\mathrm{T}_{\text {air }}$ tended to be than $\mathrm{T}_{\text {leaf }}$ from 11.30 onwards. On the two days of observation in the cool season, $\mathrm{T}_{\text {leaf }}$ tended to be warmer than $\mathrm{T}_{\text {air }}$ throughout the day (Figure 3I-J). In the hot season, $\mathrm{T}_{\text {air }}$ tended to be warmer than $\mathrm{T}_{\text {leaf }}$ in the late afternoon from 14.30 to 18.30 (Figure $3 \mathrm{~K}-\mathrm{L}$ ). The highest $\mathrm{T}_{\text {leaf }}$ was noted in the hot season reaching the maximum temperatures of $41.00-44.30{ }^{\circ} \mathrm{C}$ during 10:30-12:30 (Table 1). It is worth noting that both $\mathrm{T}_{\text {air }}$ and $\mathrm{T}_{\text {leaf }}$ were significantly higher $(p<0.001)$ in the hot season compared to the others. The mean daily $\mathrm{T}_{\text {leaf }}$ across genotypes $\left(34.48^{\circ} \mathrm{C}\right)$ in the hot was significantly higher $(p<0.001)$ than those in the rainy $\left(31.51^{\circ} \mathrm{C}\right)$ and $\operatorname{cool}\left(27.70^{\circ} \mathrm{C}\right)$ seasons (Table 1$)$. Nevertheless, in any season, no significant differences in $\mathrm{T}_{\text {leaf }}$ were found among genotypes. As shown in Table 1 , the highest mean relative humidity in the canopy $\left(\mathrm{RH}_{\mathrm{C}}\right)$ across genotypes and air relative humidity $\left(\mathrm{RH}_{\text {air }}\right)$ were recorded in rainy season with the mean daily values of 61 and $77 \%$ which differed significantly $(p<0.05)$. Similarly, in the cool season, $\mathrm{RH}_{\mathrm{C}}(29 \%)$ was also significantly lower $(p<0.05)$ than $\mathrm{RH}_{\text {air }}(42 \%)$. On the contrary, in the hot season, mean daily $\mathrm{RH}_{\mathrm{C}}(39 \%)$ was higher than $\mathrm{RH}_{\text {air }}(37 \%)$, although the difference was not significant. As shown in Figure $3 \mathrm{~S}-\mathrm{X}, \mathrm{VPD}_{\mathrm{L}}$ and $\mathrm{VPD}_{\text {air }}$ were low (less than $2 \mathrm{kPa}$ ) in the morning, increased several fold during early afternoon (particularly in the cool and hot seasons), and declined slowly in the late afternoon. As shown in Table 1, the mean daily VPD air in the hot season $(3.97 \mathrm{kPa})$ was significantly higher $(p<0.05)$ than the other seasons, and was approximately 4.0 and 1.8 fold higher than those in the rainy $(0.99 \mathrm{kPa})$ and cool $(2.19 \mathrm{kPa})$ seasons. The mean daily $\mathrm{VPD}_{\mathrm{L}}$ across genotypes in the cool $(2.45 \mathrm{kPa})$ and hot $(2.77 \mathrm{kPa})$ seasons were significantly higher than $(p<0.001)$ and approximately double that in the rainy season $(1.07 \mathrm{kPa})$. It is worth noted that the mean daily $\mathrm{VPD}_{\mathrm{L}}$ was similar to $\mathrm{VPD}_{\text {air }}$ in the rainy and cool seasons, but in the hot season $\mathrm{VPD}_{\mathrm{L}}$ was significantly lower $(p<0.05)$ than $\operatorname{VPD}_{\text {air }}$. 


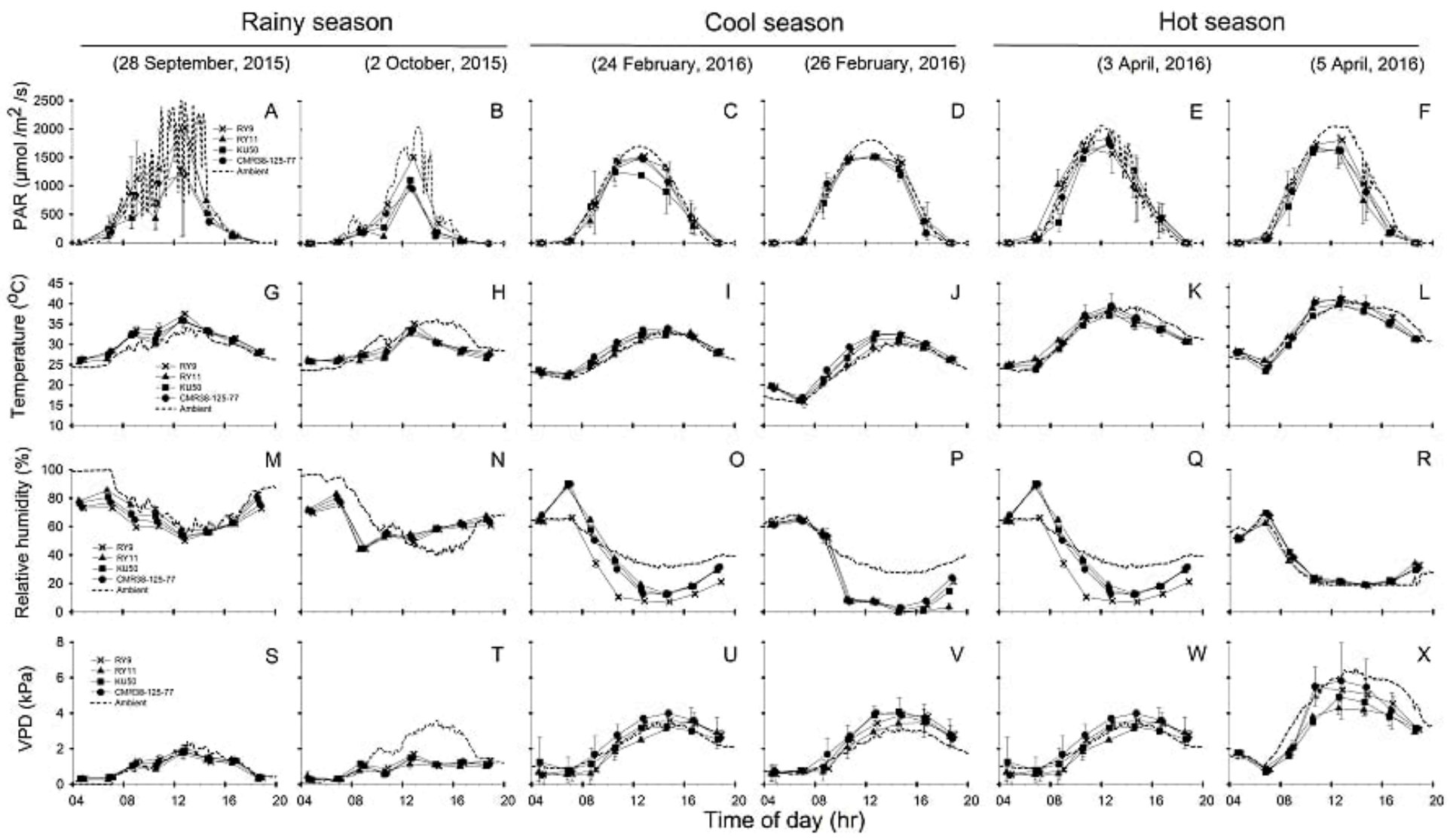

Figure 3. Diurnal variation in physical parameters including $\operatorname{PAR}_{\text {leaf }}(\mathbf{A}-\mathbf{F}), \mathrm{T}_{\text {leaf }}(\mathbf{G}-\mathbf{L}), \mathrm{RH}_{\mathrm{C}}(\mathbf{M}-\mathbf{R})$ and $\operatorname{VPD}_{\mathrm{L}}(\mathbf{S}-\mathbf{X})$ of cassava belonging to four cultivars $(\mathbf{X}, \mathrm{RY}$; $\boldsymbol{\Delta}$, RY11; $\mathbf{\square}$, KU50 and $\bullet$, CMR38-125-77) in comparison with ambient conditions ( $\mathrm{PAR}_{\mathrm{A}}, \mathrm{T}_{\text {air }}, \mathrm{RH}_{\text {air }}$ and $\left.\mathrm{VPD}_{\text {air }}\right)$ in rainy $(\mathbf{A}, \mathbf{B}, \mathbf{G}, \mathbf{H}, \mathbf{M}, \mathbf{N}, \mathbf{S}, \mathbf{T}), \operatorname{cool}(\mathbf{C}, \mathbf{D}, \mathbf{I}, \mathbf{J}, \mathbf{O}, \mathbf{P}, \mathbf{U}, \mathbf{V})$, and hot season $(\mathbf{E}, \mathbf{F}, \mathbf{K}, \mathbf{L}, \mathbf{Q}, \mathbf{R}, \mathbf{W}, \mathbf{X})$. The measurement was performed on two sunny days in each season. Leaf parameters were obtained from leaf gas exchange measurements while the environmental parameters were from the weather station. Data shows mean of two replicates \pm SD. 
Table 1. Seasonal variation in environmental and leaf/canopy parameters during the time of photosynthesis measurements i.e., two days in rainy season (25 Nov and

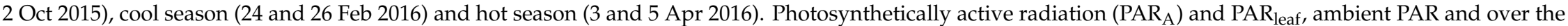
leaf surface; $\mathrm{T}_{\text {air }}$ and $\mathrm{T}_{\text {leaf }}$, ambient air temperature and leaf temperature; $\mathrm{RH}_{\text {air }}$ and $\mathrm{RH}_{\mathrm{C}}$, ambient air relative humidity ( $\mathrm{RH}$ ) and $\mathrm{RH}$ in the plant canopy; $\mathrm{VPD}$ air and $\mathrm{VPD}_{\mathrm{L}}$, ambient vapor pressure deficit and VPD of the leaf. Daily minima (min), maxima (max) and means for each genotype were from 24 measurements (six time points during 6:30-16:30 $\mathrm{h} x$ two leaves $\mathrm{x}$ two days). Means which are significantly different among seasons are denoted with capital letters. Mean of leaf parameter across genotypes which is significantly different from that of the environment is denoted with *.

\begin{tabular}{|c|c|c|c|c|c|c|c|c|c|c|c|c|}
\hline \multirow{2}{*}{ Parameter } & \multirow{2}{*}{ Genotype } & \multicolumn{3}{|c|}{ Rainy } & \multicolumn{3}{|c|}{ Cool } & \multicolumn{3}{|c|}{ Hot } & \multirow{2}{*}{$\begin{array}{c}F \text {-Test } \\
\text { Value }^{2}\end{array}$} & \multirow{2}{*}{$\frac{\text { Critical } I}{\text { Value }^{2}}$} \\
\hline & & $\min$ & $\max$ & mean & $\min$ & $\max$ & mean & $\min$ & $\max$ & mean & & \\
\hline $\operatorname{PAR}_{\mathrm{A}}\left(\mu \mathrm{mol} / \mathrm{m}^{2} / \mathrm{s}\right)$ & & 35 & 2498 & 704 & 26 & 1794 & 950 & 116 & 2038 & 1105 & 2.184 & 0.12 \\
\hline \multirow{7}{*}{$\mathrm{PAR}_{\text {leaf }}\left(\mu \mathrm{mol} / \mathrm{m}^{2} / \mathrm{s}\right)$} & RY9 & 27 & 2115 & 678 & 28 & 1528 & 909 & 75 & 1812 & 974 & 1.426 & 0.247 \\
\hline & RY11 & 12 & 2002 & $497 \mathrm{~B}$ & 6 & 1517 & $877 \mathrm{AB}$ & 108 & 1868 & $956 \mathrm{~A}$ & 3.822 & 0.027 \\
\hline & KU50 & 17 & 2114 & $423 \mathrm{~B}$ & 12 & 1521 & $800 \mathrm{AB}$ & 41 & 1817 & $875 \mathrm{~A}$ & 4.34 & 0.017 \\
\hline & CMR38-125-77 & 25 & 1964 & $473 \mathrm{~B}$ & 44 & 1516 & $875 \mathrm{AB}$ & 61 & 1777 & $913 \mathrm{~A}$ & 4.339 & 0.017 \\
\hline & $F$-test value ${ }^{1}$ & & & 0.947 & & & 0.148 & & & 0.11 & & \\
\hline & Critical- $P$ value $^{1}$ & & & 0.421 & & & 0.931 & & & 0.954 & & \\
\hline & mean & 20 & 2049 & $518 \mathrm{~B}$ & 23 & 1521 & $863 \mathrm{~A}$ & 71 & 1819 & $930 \mathrm{~A}$ & 13.385 & $<0.001$ \\
\hline $\mathrm{T}_{\text {air }}\left({ }^{\circ} \mathrm{C}\right)$ & & 25.10 & 33.00 & $28.68 \mathrm{~B}$ & 16.00 & 32.80 & $26.44 \mathrm{~B}$ & 24.40 & 40.30 & $35.04 \mathrm{~A}$ & 22.687 & $<0.001$ \\
\hline \multirow[t]{7}{*}{$\mathrm{T}_{\text {leaf }}\left({ }^{\circ} \mathrm{C}\right)$} & RY9 & 26.45 & 37.78 & $31.26 \mathrm{~B}$ & 14.85 & 33.61 & $28.05 \mathrm{~B}$ & 25.34 & 42.52 & $35.00 \mathrm{~A}$ & 12.796 & $<0.001$ \\
\hline & RY11 & 25.60 & 36.06 & $29.81 \mathrm{C}$ & 15.71 & 33.02 & $26.57 \mathrm{~B}$ & 26.14 & 41.12 & $35.55 \mathrm{~A}$ & 19.516 & $<0.001$ \\
\hline & KU50 & 25.80 & 37.15 & $30.68 \mathrm{AB}$ & 16.02 & 32.94 & $27.48 \mathrm{~B}$ & 23.24 & 41.00 & $33.45 \mathrm{~A}$ & 9.703 & $<0.001$ \\
\hline & CMR38-125-77 & 26.21 & 37.09 & $31 \mathrm{~B}$ & 16.66 & 33.99 & $28.69 \mathrm{~B}$ & 24.26 & 44.30 & $34.90 \mathrm{~A}$ & 10.311 & $<0.001$ \\
\hline & $F$-test value ${ }^{1}$ & & & 0.92 & & & 0.729 & & & 0.417 & & \\
\hline & Critical- $P$ value ${ }^{1}$ & & & 0.434 & & & 0.538 & & & 0.741 & & \\
\hline & mean & 26.02 & 37.02 & $31.51 * \mathrm{~B}$ & 15.81 & 33.39 & $27.70 \mathrm{C}$ & 24.75 & 42.24 & $34.48 \mathrm{~A}$ & 41.177 & $<0.001$ \\
\hline $\mathrm{RH}_{\text {air }}(\%)$ & & 59 & 100 & $77 \mathrm{~A}$ & 27 & 68 & $42 \mathrm{~B}$ & 19 & 82 & $37 \mathrm{~B}$ & 45.802 & $<0.001$ \\
\hline \multirow[t]{7}{*}{$\mathrm{RH}_{\mathrm{C}}(\%)$} & RY9 & 45 & 76 & $59 \mathrm{~A}$ & 1 & 66 & $23 \mathrm{C}$ & 19 & 75 & $38 \mathrm{~B}$ & 23.805 & $<0.001$ \\
\hline & RY11 & 44 & 86 & $64 \mathrm{~A}$ & 1 & 88 & $32 \mathrm{~B}$ & 19 & 73 & $37 \mathrm{~B}$ & 16.350 & $<0.001$ \\
\hline & KU50 & 45 & 82 & $62 \mathrm{~A}$ & 1 & 90 & $30 \mathrm{~B}$ & 19 & 84 & $41 \mathrm{~B}$ & 13.872 & $<0.001$ \\
\hline & CMR38-125-77 & 45 & 78 & $60 \mathrm{~A}$ & 3 & 90 & $30 \mathrm{~B}$ & 19 & 78 & $40 \mathrm{~B}$ & 14.917 & $<0.001$ \\
\hline & $F$-test value ${ }^{1}$ & & & 0.975 & & & 0.49 & & & 0.201 & & \\
\hline & Critical- $P$ value ${ }^{1}$ & & & 0.408 & & & 0.69 & & & 0.895 & & \\
\hline & mean & 45 & 81 & $61 * \mathrm{~A}$ & 2 & 84 & $29 * C$ & 19 & 78 & $39 \mathrm{~B}$ & 67.503 & $<0.001$ \\
\hline
\end{tabular}


Table 1. Cont.

\begin{tabular}{|c|c|c|c|c|c|c|c|c|c|c|c|c|}
\hline \multirow{2}{*}{ Parameter } & \multirow{2}{*}{ Genotype } & \multicolumn{3}{|c|}{ Rainy } & \multicolumn{3}{|c|}{ Cool } & \multicolumn{3}{|c|}{ Hot } & \multirow{2}{*}{$\begin{array}{c}F \text {-Test } \\
\text { Value }^{2}\end{array}$} & \multirow{2}{*}{$\begin{array}{c}\text { Critical } I \\
\text { Value }^{2}\end{array}$} \\
\hline & & $\min$ & $\max$ & mean & $\min$ & $\max$ & mean & $\min$ & $\max$ & mean & & \\
\hline $\mathrm{VPD}_{\text {air }}(\mathrm{kPa})$ & & 0.01 & 2.07 & $0.99 \mathrm{C}$ & 0.59 & 3.37 & $2.19 \mathrm{~B}$ & 0.55 & 6.2 & $3.97 \mathrm{~A}$ & 33.359 & $<0.001$ \\
\hline \multirow[t]{7}{*}{$\operatorname{VPD}_{\mathrm{L}}(\mathrm{kPa})$} & RY9 & 0.28 & 2.14 & $1.17 \mathrm{~B}$ & 0.3 & 4.04 & $2.47 \mathrm{~A}$ & 0.41 & 5.61 & $2.94 \mathrm{~A}$ & 12.421 & $<0.001$ \\
\hline & RY11 & 0.23 & 1.76 & $0.94 \mathrm{~B}$ & 0.2 & 4.08 & $2.12 \mathrm{~A}$ & 0.49 & 4.53 & $2.51 \mathrm{~A}$ & 14.424 & $<0.001$ \\
\hline & KU50 & 0.26 & 2.08 & $1.08 \mathrm{~B}$ & 0.3 & 4.65 & $2.46 \mathrm{~A}$ & 0.16 & 5.15 & $2.47 \mathrm{~A}$ & 11.041 & $<0.001$ \\
\hline & CMR38-125-77 & 0.28 & 2.09 & $1.10 \mathrm{~B}$ & 0.6 & 4.15 & $2.76 \mathrm{~A}$ & 0.24 & 7.33 & $3.15 \mathrm{~A}$ & 14.401 & $<0.001$ \\
\hline & $F$-test value ${ }^{1}$ & & & 1.061 & & & 1.000 & & & 0.99 & & \\
\hline & Critical- $P$ value $^{1}$ & & & 0.37 & & & 0.397 & & & 0.401 & & \\
\hline & mean & 0.26 & 2.02 & $1.07 \mathrm{~B}$ & 0.35 & 4.23 & $2.45 \mathrm{~A}$ & 0.33 & 5.66 & $2.77 * \mathrm{~A}$ & 51.156 & $<0.001$ \\
\hline
\end{tabular}

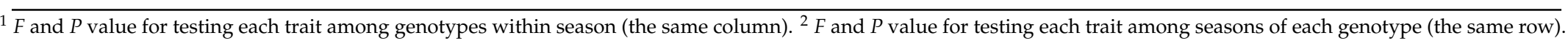




\subsection{Diurnal Chl Fluorescence of Cassava Leaves}

Diurnal patterns of Chl fluorescence parameters ( $\Phi_{\mathrm{PSII}}$, ETR and NPQ) of four cassava genotypes measured during 04:30-18:30 on two sunny days in each of the three seasons were depicted in Figure 4 . For each season, the diurnal minima, maxima and means of $\Phi_{\text {PSII }}$, ETR and NPQ recorded during 06:30 to 16:30 across two days of investigation were summarized in Table 2. The values for $F_{v} / F_{m}$ were obtained from the measurements in the dark at 04:30.

The $\mathrm{F}_{\mathrm{v}} / \mathrm{F}_{\mathrm{m}}$ values were high for all genotypes and in all seasons, although some significant differences were detected. The $\mathrm{F}_{\mathrm{v}} / \mathrm{F}_{\mathrm{m}}$ means among genotypes were significantly different $(p<0.001)$ only in the rainy season (Table 2$)$ with RY9 showing the highest $F_{v} / F_{m}(0.866)$ which was significantly higher $(p<0.001)$ than RY11 $(0.845)$ but not different from that of KU50 (0.847) and CMR38-125-77 (0.858). Seasonal variation in $\mathrm{F}_{\mathrm{v}} / \mathrm{F}_{\mathrm{m}}$ was observed i.e., the means across genotypes in rainy $(0.854)$ and hot $(0.849)$ seasons were significantly higher $(p<0.001)$ than that in the cool $(0.838)$ season (Table 2$)$.

Diurnal patterns of $\Phi_{\mathrm{PSII}}$ displayed the inverted bell-shaped curves, and in the cool and hot seasons, $\Phi_{\mathrm{PSII}}$ values during 6:30 to 12:30 tended to decrease more rapidly than those in the rainy season (Figure $4 \mathrm{~A}-\mathrm{F})$. The differences in $\Phi_{\mathrm{PSII}}$ means across genotypes were noted among seasons being significantly $(p<0.001)$ higher $(0.70)$ in the rainy than the hot $(0.58)$ and cool $(0.56)$ seasons (Table 2). However, no significance differences were found among genotypes in any season. Changes in ETR over the course of the day (Figure 4G-L) were related to the intensity of sunlight (Figure 3A-F). As shown in Table 2, daily means for ETR across genotypes in the hot $\left(159 \mu \mathrm{mol}\left(\mathrm{e}^{-}\right) / \mathrm{m}^{2} / \mathrm{s}\right)$ and cool $\left(157 \mu \mathrm{mol}\left(\mathrm{e}^{-}\right) / \mathrm{m}^{2} / \mathrm{s}\right)$ were significantly higher $(p<0.001)$ than that in the rainy $\left(90 \mu \mathrm{mol}\left(\mathrm{e}^{-}\right) / \mathrm{m}^{2} / \mathrm{s}\right)$ season. However, no significance differences in mean ETR were found among genotypes in any season. Diurnal patterns of NPQ were similar to those of ETR (Figure 4M-R). Cassava genotypes exhibited highest mean NPQ across genotypes (0.45) in the hot season which was significantly different $(p<0.001)$ from that in the rainy season (0.34), and expressed an intermediate value (0.41) in the cool season (Table 2). In any season, mean NPQ among genotypes did not differ significantly. However, it was noted that NPQ of CMR-38-125-77 tended to be higher than those of the other genotypes during 10:30 to $14: 30$ in the hot season (Figure $4 \mathrm{Q}, \mathrm{R}$ ). 


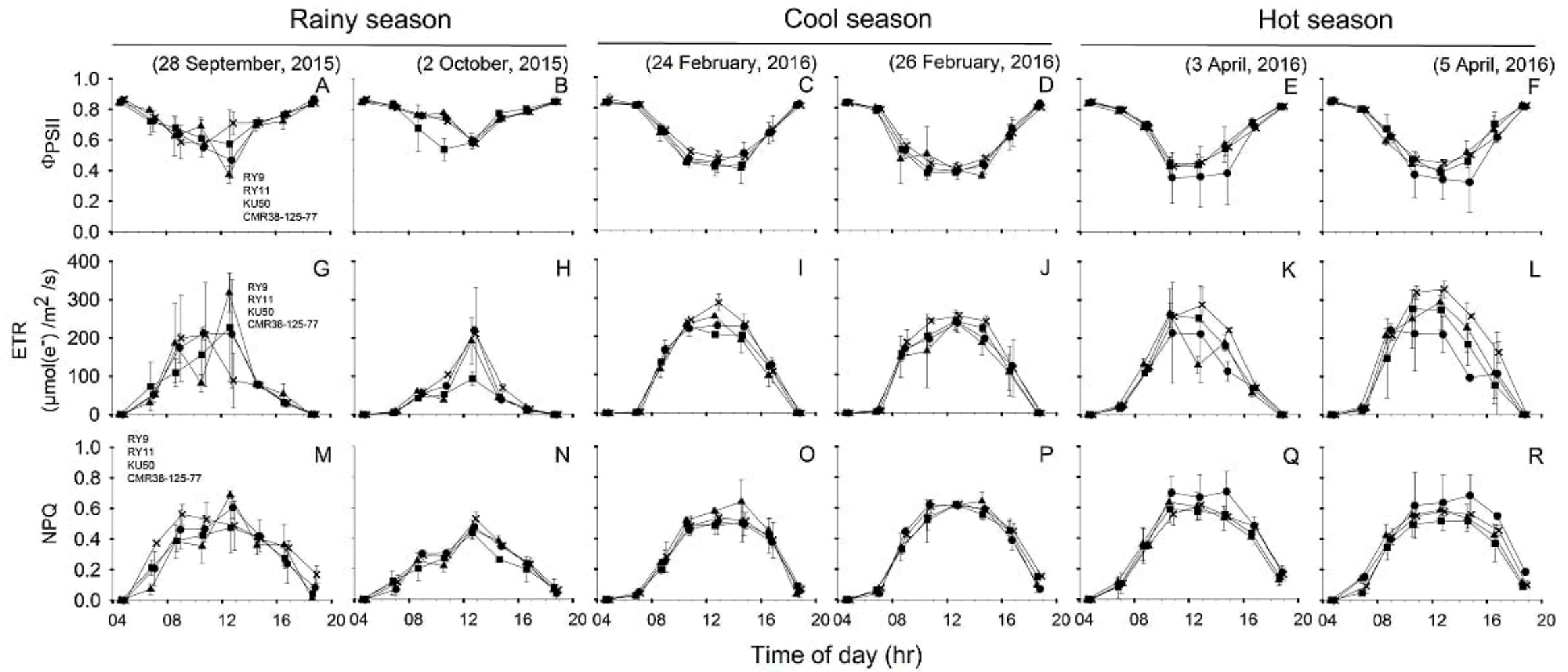

Figure 4. Diurnal pattern of Chl fluorescence parameters of four cassava genotypes (X, RY9; $\boldsymbol{\Delta}, \mathrm{RY} 11$; $\boldsymbol{\mathbf { Q }}$, KU50 and $\boldsymbol{\bullet}$, CMR38-125-77). Effective quantum yield of PSII photochemistry $\left(\Phi_{\mathrm{PSII}}\right)(\mathbf{A}-\mathbf{F})$, electron transport rate $(\mathrm{ETR})(\mathbf{G}-\mathbf{L})$ and non-photochemical quenching (NPQ) (M-R). The measurements were performed from 4:30 to 18:30 at 2-h intervals on two sunny days in rainy $(\mathbf{A}, \mathbf{B}, \mathbf{G}, \mathbf{H}, \mathbf{M}, \mathbf{N})$, cool $(\mathbf{C}, \mathbf{D}, \mathbf{I}, \mathbf{J}, \mathbf{O}, \mathbf{P})$, and hot $(\mathbf{E}, \mathbf{F}, \mathbf{K}, \mathbf{L}, \mathbf{Q}, \mathbf{R})$ season. Data shows mean of two replicates \pm SD. 
Table 2. Seasonal variation in Chl fluorescence and leaf gas exchange parameters of four cassava genotypes. $\mathrm{F}_{\mathrm{v}} / \mathrm{F}_{\mathrm{m}}$, maximal photochemical quantum yield of PSII; $\Phi_{\text {PSII }}$, effective quantum yield of PSII photochemistry; ETR, electron transfer rate; NPQ, non-photochemical quenching; R, respiration rate; Pn; net photosynthetic rate; $\mathrm{Gs}$, stomatal conductance; $\mathrm{Tr}$, transpiration rate; $\mathrm{Ci}$, intercellular $\mathrm{CO}_{2}$ concentration; $\mathrm{Ci} / \mathrm{Ca}$, ratio between intercellular and ambient $\mathrm{CO}_{2}$ concentration; $\mathrm{LUE}$, light use efficiency; WUE, water use efficiency. Minima (min), maxima (max) and means were from 24 measurements (six time points during 6:30-16:30 h x two leaves x two days in each season), except the values for $F_{v} / F_{m}$ and $R$ in which 4 measurements were taken from two leaves of each genotype at 04:30 on two days. Means which are significantly different $(p<0.05)$ among genotypes are denoted with different lower case letters, whereas those among seasons are represented by different capital letters.

\begin{tabular}{|c|c|c|c|c|c|c|c|c|c|c|c|c|}
\hline \multirow{2}{*}{ Parameter } & \multirow{2}{*}{ Genotype } & \multicolumn{3}{|c|}{ Rainy } & \multicolumn{3}{|c|}{ Cool } & \multicolumn{3}{|c|}{ Hot } & \multirow{2}{*}{$\begin{array}{c}\text { F-test } \\
\text { Value }^{2}\end{array}$} & \multirow{2}{*}{$\frac{\text { Critical-I }}{\text { Value }^{2}}$} \\
\hline & & $\min$ & $\max$ & mean & $\min$ & $\max$ & mean & $\min$ & $\max$ & mean & & \\
\hline \multirow{5}{*}{$\mathrm{F}_{\mathrm{v}} / \mathrm{F}_{\mathrm{m}}$} & RY9 & 0.862 & 0.871 & $0.866 \mathrm{a}$ & 0.823 & 0.878 & 0.845 & 0.848 & 0.856 & 0.852 & 2.681 & 0.122 \\
\hline & RY11 & 0.842 & 0.849 & $0.845 \mathrm{~b}$ & 0.828 & 0.836 & 0.833 & 0.829 & 0.862 & 0.845 & 2.765 & 0.116 \\
\hline & KU50 & 0.844 & 0.85 & $0.847 \mathrm{ab} \mathrm{A}$ & 0.829 & 0.846 & $0.837 \mathrm{~B}$ & 0.842 & 0.854 & $0.847 \mathrm{~A}$ & 4.915 & 0.036 \\
\hline & CMR38-125-77 & 0.85 & 0.866 & $0.858 \mathrm{ab} \mathrm{A}$ & 0.833 & 0.843 & $0.838 \mathrm{~B}$ & 0.848 & 0.862 & $0.854 \mathrm{~A}$ & 9.950 & 0.005 \\
\hline & $F$-test value ${ }^{1}$ & & & 15.126 & & & 0.374 & & & 0.974 & & \\
\hline \multirow{8}{*}{$\Phi_{\mathrm{PSII}}$} & Critical- $P$ value ${ }^{1}$ & & & $<0.001$ & & & 0.773 & & & 0.437 & & \\
\hline & mean & & & $0.854 \mathrm{~A}$ & & & $0.838 \mathrm{~B}$ & & & $0.849 \mathrm{~A}$ & 11.142 & $<0.001$ \\
\hline & RY9 & 0.51 & 0.81 & $0.71 \mathrm{~A}$ & 0.41 & 0.82 & $0.58 \mathrm{~B}$ & 0.43 & 0.81 & $0.59 \mathrm{~B}$ & 8.852 & $<0.001$ \\
\hline & RY11 & 0.34 & 0.82 & $0.70 \mathrm{~A}$ & 0.33 & 0.82 & $0.54 \mathrm{~B}$ & 0.38 & 0.8 & $0.59 \mathrm{AB}$ & 7.105 & 0.002 \\
\hline & KU50 & 0.41 & 0.85 & $0.69 \mathrm{~A}$ & 0.37 & 0.82 & $0.54 \mathrm{~B}$ & 0.35 & 0.81 & $0.60 \mathrm{AB}$ & 6.476 & 0.003 \\
\hline & CMR38-125-77 & 0.39 & 0.82 & $0.69 \mathrm{~A}$ & 0.35 & 0.83 & $0.56 \mathrm{~B}$ & 0.19 & 0.8 & $0.53 \mathrm{~B}$ & 6.850 & 0.002 \\
\hline & $F$-test value ${ }^{1}$ & & & 0.135 & & & 0.250 & & & 1.059 & & \\
\hline & Critical- $P$ value ${ }^{1}$ & & & 0.939 & & & 0.861 & & & 0.352 & & \\
\hline \multirow{4}{*}{$\begin{array}{c}\text { ETR } \\
{\left[\mu \mathrm{mol}(\mathrm{e}-) / \mathrm{m}^{2} / \mathrm{s}\right]}\end{array}$} & mean & & & $0.70 \mathrm{~A}$ & & & $0.56 \mathrm{~B}$ & & & $0.58 \mathrm{~B}$ & 27.745 & $<0.001$ \\
\hline & RY9 & 8 & 304 & $94 \mathrm{~B}$ & 2 & 304 & $173 \mathrm{~A}$ & 16 & 343 & $190 \mathrm{~A}$ & 6.758 & 0.002 \\
\hline & RY11 & 5 & 353 & $92 \mathrm{~B}$ & 1 & 257 & $144 \mathrm{AB}$ & 19 & 310 & $158 \mathrm{~A}$ & 3.351 & 0.041 \\
\hline & KU50 & 4 & 327 & $77 \mathrm{~B}$ & 1 & 260 & $152 \mathrm{~A}$ & 11 & 309 & $155 \mathrm{~A}$ & 6.325 & 0.003 \\
\hline \multirow{11}{*}{ NPQ } & CMR38-125-77 & 6 & 310 & 97 & 2 & 256 & 158 & 14 & 307 & 134 & 3.079 & 0.052 \\
\hline & $F$-test value ${ }^{1}$ & & & 0.252 & & & 0.481 & & & 1.300 & & \\
\hline & Critical- $P$ value ${ }^{1}$ & & & 0.860 & & & 0.696 & & & 0.279 & & \\
\hline & mean & & & $90 \mathrm{~B}$ & & & $157 \mathrm{~A}$ & & & $159 \mathrm{~A}$ & 18.251 & $<0.001$ \\
\hline & RY9 & 0.08 & 0.61 & 0.37 & 0.04 & 0.64 & 0.42 & 0.06 & 0.64 & 0.44 & 0.956 & 0.389 \\
\hline & RY11 & 0.05 & 0.71 & 0.32 & 0.01 & 0.74 & 0.42 & 0.09 & 0.67 & 0.44 & 2.825 & 0.066 \\
\hline & KU50 & 0.12 & 0.59 & 0.31 & 0.02 & 0.63 & 0.39 & 0.03 & 0.65 & 0.41 & 2.36 & 0.102 \\
\hline & CMR38-125-77 & 0.04 & 0.66 & $0.34 \mathrm{~B}$ & 0.03 & 0.65 & $0.40 \mathrm{AB}$ & 0.06 & 0.8 & $0.50 \mathrm{~A}$ & 4.255 & 0.018 \\
\hline & $F$-test value ${ }^{1}$ & & & 0.951 & & & 0.128 & & & 1.100 & & \\
\hline & Critical- $P$ value ${ }^{1}$ & & & 0.419 & & & 0.943 & & & 0.353 & & \\
\hline & mean & & & $0.34 \mathrm{~B}$ & & & $0.41 \mathrm{~A}$ & & & $0.45 \mathrm{~A}$ & 9.55 & $<0.001$ \\
\hline
\end{tabular}


Table 2. Cont.

\begin{tabular}{|c|c|c|c|c|c|c|c|c|c|c|c|c|}
\hline \multirow{2}{*}{ Parameter } & \multirow{2}{*}{ Genotype } & \multicolumn{3}{|c|}{ Rainy } & \multicolumn{3}{|c|}{ Cool } & \multicolumn{3}{|c|}{ Hot } & \multirow{2}{*}{$\frac{F \text {-test }}{\text { Value }^{2}}$} & \multirow{2}{*}{$\begin{array}{c}\text { Critical- } P \\
\text { Value }^{2}\end{array}$} \\
\hline & & $\min$ & $\max$ & mean & $\min$ & $\max$ & mean & $\min$ & $\max$ & mean & & \\
\hline $\mathrm{R}$ & RY9 & 1.27 & 1.94 & $1.63 \mathrm{~B}$ & 0.85 & 1.7 & $1.26 \mathrm{ab} \mathrm{B}$ & 2.25 & 3.03 & 2.61 a A & 14.37 & 0.002 \\
\hline \multirow{6}{*}[\mu\mathrm{molCO}_{2}/\mathrm{m}^{2}/\mathrm{s}]{} & RY11 & 1.18 & 2.41 & $1.65 \mathrm{AB}$ & 1.13 & 1.91 & $1.52 \mathrm{ab} B$ & 2.23 & 2.64 & $2.39 \mathrm{ab} \mathrm{A}$ & 5.296 & 0.03 \\
\hline & KU50 & 0.74 & 1.81 & $1.38 \mathrm{AB}$ & 0.68 & 1.05 & $0.84 \mathrm{~b} \mathrm{~B}$ & 1.3 & 2.32 & $1.82 \mathrm{~b} \mathrm{~A}$ & 6.599 & 0.017 \\
\hline & CMR38-125-77 & 0.97 & 1.58 & $1.23 \mathrm{~B}$ & 1.59 & 4.26 & 2.96 a A & 1.95 & 2.26 & $2.14 \mathrm{ab} A B$ & 6.164 & 0.021 \\
\hline & $F$-test value ${ }^{1}$ & & & 1.048 & & & 7.544 & & & 4.95 & & \\
\hline & Critical- $P$ value ${ }^{1}$ & & & 0.407 & & & 0.004 & & & 0.018 & & \\
\hline & mean & & & $1.47 \mathrm{~B}$ & & & $1.65 \mathrm{~B}$ & & & $2.24 \mathrm{~A}$ & 5.701 & 0.006 \\
\hline Pn & RY9 & 0.20 & 36.00 & 14.00 & 0.78 & 32.02 & 16.77 & 1.33 & 31.57 & 14.67 & 0.895 & 0.413 \\
\hline \multirow{6}{*}{$\left(\mu \mathrm{molCO}{ }_{2} / \mathrm{m}^{2} / \mathrm{s}\right)$} & RY11 & 0.70 & 31.70 & 11.20 & 0.72 & 30.09 & 17.19 & 1.81 & 33.31 & 17.02 & 2.036 & 0.138 \\
\hline & KU50 & 0.10 & 28.40 & 9.54 & 0.22 & 23.14 & 11.77 & 0.1 & 31.78 & 14.64 & 1.835 & 0.167 \\
\hline & CMR38-125-77 & 0.90 & 27.50 & 12.30 & 0.46 & 26.51 & 12.86 & 0.14 & 31.99 & 10.97 & 0.281 & 0.756 \\
\hline & $F$-test value ${ }^{1}$ & & & 0.397 & & & 1.51 & & & 1.471 & & \\
\hline & Critical- $P$ value $^{1}$ & & & 0.755 & & & 0.217 & & & 0.228 & & \\
\hline & mean & & & $11.75 \mathrm{~B}$ & & & $14.60 \mathrm{~A}$ & & & $14.32 \mathrm{AB}$ & 3.157 & 0.044 \\
\hline Gs & RY9 & 0.10 & 1.24 & $0.58 \mathrm{~A}$ & 0.01 & 0.57 & $0.23 \mathrm{~B}$ & 0.05 & 0.59 & $0.27 \mathrm{~B}$ & 18.141 & $<0.001$ \\
\hline \multirow{6}{*}{$\left(\mathrm{molH}_{2} \mathrm{O} / \mathrm{m}^{2} / \mathrm{s}\right)$} & RY11 & 0.11 & 1.71 & $0.57 \mathrm{~A}$ & 0.05 & 0.59 & $0.26 \mathrm{~B}$ & 0.08 & 0.69 & $0.40 \mathrm{~A}$ & 9.266 & $<0.001$ \\
\hline & KU50 & 0.08 & 1.97 & $0.53 \mathrm{~A}$ & 0.00 & 0.41 & $0.18 \mathrm{~B}$ & 0.05 & 0.75 & $0.34 \mathrm{~A}$ & 8.253 & $<0.001$ \\
\hline & CMR38-125-77 & 0.02 & 1.03 & $0.44 \mathrm{~A}$ & 0.01 & 0.36 & $0.17 \mathrm{~B}$ & 0.02 & 1.11 & $0.26 \mathrm{~B}$ & 9.397 & $<0.001$ \\
\hline & $F$-test value ${ }^{1}$ & & & 0.67 & & & 1.525 & & & 2.156 & & \\
\hline & Critical- $P$ value $^{1}$ & & & 0.572 & & & 0.213 & & & 0.099 & & \\
\hline & mean & & & $0.53 \mathrm{~A}$ & & & $0.21 \mathrm{C}$ & & & $0.32 \mathrm{~B}$ & 40.295 & $<0.001$ \\
\hline $\operatorname{Tr}$ & RY9 & 1.43 & 10.33 & 5.28 & 0.09 & 12.87 & 5.36 & 0.74 & 11.26 & $6.36 \mathrm{ab}$ & 0.730 & 0.486 \\
\hline \multirow{6}{*}{$\mathrm{mmolH}_{2} \mathrm{O} / \mathrm{m}^{2} / \mathrm{s}$} & RY11 & 0.75 & 8.76 & $4.23 \mathrm{~B}$ & 0.07 & 13.33 & $5.15 \mathrm{~B}$ & 1.34 & 15.22 & 8.52 a A & 8.695 & $<0.001$ \\
\hline & KU50 & 1.02 & 8.95 & $4.08 \mathrm{AB}$ & 0.03 & 11.13 & $3.88 \mathrm{~B}$ & 0.34 & 12.09 & $6.74 \mathrm{ab} A$ & 6.484 & 0.003 \\
\hline & CMR38-125-77 & 1.11 & 8.68 & 4.52 & 0.1 & 9.44 & 4.24 & 0.53 & 11.75 & $4.74 \mathrm{~b}$ & 0.149 & 0.862 \\
\hline & $F$-test value ${ }^{1}$ & & & 1.074 & & & 0.868 & & & 3.719 & & \\
\hline & Critical- $P$ value ${ }^{1}$ & & & 0.364 & & & 0.461 & & & 0.014 & & \\
\hline & mean & & & $4.52 \mathrm{~B}$ & & & $4.65 \mathrm{~B}$ & & & $6.58 \mathrm{~A}$ & 11.227 & $<0.001$ \\
\hline $\mathrm{Ci}$ & RY9 & 183 & 436 & $328 \mathrm{~A}$ & 104 & 548 & $250 \mathrm{~B}$ & 137 & 455 & $267 \mathrm{~B}$ & 4.663 & 0.013 \\
\hline \multirow{6}{*}{$\left(\mu \mathrm{molCO}{ }_{2} / \mathrm{mol}\right.$ air $]$} & RY11 & 198 & 493 & 338 & 97 & 563 & 275 & 201 & 433 & 292 & 2.967 & 0.058 \\
\hline & KU50 & 215 & 482 & $341 \mathrm{~A}$ & 107 & 504 & $257 \mathrm{~B}$ & 190 & 476 & $300 \mathrm{AB}$ & 5.795 & 0.005 \\
\hline & CMR38-125-77 & 213 & 443 & $334 \mathrm{~A}$ & 92 & 579 & $252 \mathrm{~B}$ & 96 & 468 & $265 \mathrm{~B}$ & 4.731 & 0.012 \\
\hline & $F$-test value ${ }^{1}$ & & & 0.131 & & & 0.243 & & & 0.961 & & \\
\hline & Critical- $P$ value $^{1}$ & & & 0.941 & & & 0.866 & & & 0.415 & & \\
\hline & mean & & & $335 \mathrm{~A}$ & & & $258 \mathrm{~B}$ & & & $281 \mathrm{~B}$ & 17.73 & $<0.001$ \\
\hline
\end{tabular}


Table 2. Cont.

\begin{tabular}{|c|c|c|c|c|c|c|c|c|c|c|c|c|}
\hline \multirow{2}{*}{ Parameter } & \multirow{2}{*}{ Genotype } & \multicolumn{3}{|c|}{ Rainy } & \multicolumn{3}{|c|}{ Cool } & \multicolumn{3}{|c|}{ Hot } & \multirow{2}{*}{$\begin{array}{c}\text {-test } \\
\text { Value }^{2}\end{array}$} & \multirow{2}{*}{$\begin{array}{c}\text { Critical-P } \\
\text { Value }^{2}\end{array}$} \\
\hline & & $\min$ & $\max$ & mean & $\min$ & $\max$ & mean & $\min$ & $\max$ & mean & & \\
\hline \multirow[t]{7}{*}{$\mathrm{Ci} / \mathrm{Ca}$} & RY9 & 0.56 & 1.00 & $0.86 \mathrm{~A}$ & 0.28 & 1.31 & $0.64 \mathrm{~B}$ & 0.37 & 0.98 & $0.68 \mathrm{~B}$ & 7.825 & $<0.001$ \\
\hline & RY11 & 0.52 & 0.99 & $0.85 \mathrm{~A}$ & 0.26 & 1.33 & $0.69 \mathrm{~B}$ & 0.57 & 0.96 & $0.76 \mathrm{AB}$ & 4.239 & 0.018 \\
\hline & KU50 & 0.64 & 0.99 & $0.87 \mathrm{~A}$ & 0.28 & 1.20 & $0.65 \mathrm{~B}$ & 0.52 & 0.99 & $0.75 \mathrm{AB}$ & 9.781 & $<0.001$ \\
\hline & CMR38-125-77 & 0.64 & 0.99 & $0.86 \mathrm{~A}$ & 0.28 & 1.20 & $0.65 \mathrm{~B}$ & 0.25 & 0.99 & $0.67 \mathrm{~B}$ & 8.91 & $<0.001$ \\
\hline & $F$-test value ${ }^{1}$ & & & 0.0875 & & & 0.215 & & & 2.036 & & \\
\hline & Critical- $P$ value ${ }^{1}$ & & & 0.967 & & & 0.886 & & & 0.114 & & \\
\hline & mean & & & $0.85 \mathrm{~A}$ & & & $0.70 \mathrm{~B}$ & & & $0.71 \mathrm{~B}$ & 29.177 & $<0.001$ \\
\hline LUE & RY9 & 0.009 & 0.034 & $0.023 \mathrm{~b}$ & 0.016 & 0.04 & 0.025 & 0.01 & 0.035 & $0.020 \mathrm{ab}$ & 1.845 & 0.166 \\
\hline \multirow{6}{*}{ ( $\mu \mathrm{molCO}_{2} / \mu \mathrm{mol}$ photon) } & RY11 & 0.015 & 0.097 & $0.035 \mathrm{a}$ & 0.012 & 0.116 & 0.029 & 0.014 & 0.036 & $0.023 \mathrm{a}$ & 1.596 & 0.210 \\
\hline & KU50 & 0.016 & 0.056 & $0.030 \mathrm{ab} \mathrm{A}$ & 0.011 & 0.039 & $0.021 \mathrm{AB}$ & 0.002 & 0.038 & $0.020 \mathrm{ab} B$ & 3.509 & 0.035 \\
\hline & CMR38-125-77 & 0.016 & 0.058 & 0.033 a A & 0.01 & 0.035 & $0.020 \mathrm{~B}$ & 0.001 & 0.043 & $0.016 \mathrm{~b} \mathrm{~B}$ & 11.196 & $<0.001$ \\
\hline & $F$-test value ${ }^{1}$ & & & 2.791 & & & 1.592 & & & 2.871 & & \\
\hline & Critical- $P$ value ${ }^{1}$ & & & 0.045 & & & 0.197 & & & 0.041 & & \\
\hline & mean & & & $0.030 \mathrm{~A}$ & & & $0.023 \mathrm{~B}$ & & & $0.019 \mathrm{~B}$ & 9.46 & $<0.001$ \\
\hline WUE & RY9 & 0.09 & 5.26 & $2.21 \mathrm{~B}$ & 1.14 & 10.98 & $4.76 \mathrm{~A}$ & 0.84 & 3.86 & $2.26 \mathrm{~B}$ & 14.508 & $<0.001$ \\
\hline \multirow[t]{6}{*}{$\left(\mu \mathrm{molCO} / \mathrm{mmol} \mathrm{H}_{2} \mathrm{O}\right)$} & RY11 & 0.26 & 6.15 & $2.34 \mathrm{~B}$ & 0.62 & 12.36 & $4.79 \mathrm{~A}$ & 0.55 & 3.41 & $1.92 \mathrm{~B}$ & 7.374 & 0.001 \\
\hline & KU50 & 0.14 & 6.54 & $2.28 \mathrm{~B}$ & 0.48 & 13.79 & $4.30 \mathrm{~A}$ & 0.21 & 3.14 & $1.86 \mathrm{~B}$ & 7.770 & $<0.001$ \\
\hline & CMR38-125-77 & 0.37 & 5.9 & $2.52 \mathrm{~B}$ & 1.43 & 14.82 & $4.25 \mathrm{~A}$ & 0.18 & 4.64 & $2.18 \mathrm{~B}$ & 6.903 & 0.002 \\
\hline & $F$-test value ${ }^{1}$ & & & 1.118 & & & 0.299 & & & 1.135 & & \\
\hline & Critical- $P$ value ${ }^{1}$ & & & 0.346 & & & 0.826 & & & 0.339 & & \\
\hline & mean & & & $2.33 \mathrm{~B}$ & & & $4.52 \mathrm{~A}$ & & & $2.05 \mathrm{~B}$ & 26.604 & $<0.001$ \\
\hline
\end{tabular}

${ }^{1} \mathrm{~F}$ and $P$ value for testing each trait among genotypes within season (the same column). ${ }^{2} \mathrm{~F}$ and $P$ value for testing each trait among seasons of each genotype (the same row). 


\subsection{Diurnal Leaf Gas Exchange}

Diurnal responses of leaf gas exchange and related parameters, i.e., Pn, Gs and Tr of four cassava genotypes measured during 04:30-18:30 on the same days as Chl fluorescence measurements were displayed in Figure 5. For each season, the diurnal minima, maxima and means of R, Pn, Gs, Tr, Ci $\mathrm{Ci} / \mathrm{Ca}$, LUE and WUE which were recorded during 06:30 to 16:30 across two days of investigation were summarized in Table 2. The values for dark respiration rates $(R)$ in Table 2 were obtained from leaf gas exchange measurements in the dark at 04:30.

Seasonal variation in $\mathrm{R}$ of cassava leaves was observed in which the mean across genotypes was highest in the hot $\left(2.24 \mu \mathrm{molCO} / 2 / \mathrm{m}^{2} / \mathrm{s}\right)$ followed by the significantly lower rates $(p=0.006)$ in the cool $\left(1.65 \mu \mathrm{molCO} 2 / \mathrm{m}^{2} / \mathrm{s}\right)$ and the rainy $\left(1.47 \mu \mathrm{molCO}_{2} / \mathrm{m}^{2} / \mathrm{s}\right)$ seasons (Table 2$)$. Significantly different $\mathrm{R}$ among genotypes were observed in the hot and cool seasons with KU50 showing significantly lower R $(p<0.05)$ than CMR38-125-77 in the cool season, and RY9 in the hot season.

Diurnal changes in Pn (Figure 5A-F) were closely related to those of $\mathrm{PAR}_{\text {leaf }}$ (Figure 3A-F). The highest mean Pn across genotypes was found in the cool season at $14.60 \mu \mathrm{molCO} / \mathrm{C}_{2} / \mathrm{s}$ followed by that of the hot season at $14.32 \mu \mathrm{molCO} / 2 / \mathrm{m}^{2} / \mathrm{s}$. The lowest mean $\mathrm{Pn}$ across genotypes was found in the rainy season $\left(11.75 \mu \mathrm{molCO} / 2 \mathrm{~m}^{2} / \mathrm{s}\right)$ which was significantly lower $(p=0.044)$ than that in the cool $\left(14.60 \mu \mathrm{molCO} / 2 / \mathrm{m}^{2} / \mathrm{s}\right)$ season. However, Pn among the four genotypes were not significantly different in any season.

The patterns of diurnal response in Gs (Figure 5G-L) were related to variation in Pn (Figure 5A-F). Means of Gs across genotypes significantly $(p<0.001)$ differed among the three seasons being highest in the rainy $\left(0.53 \mathrm{molH}_{2} \mathrm{O} / \mathrm{m}^{2} / \mathrm{s}\right)$ followed by the hot $\left(0.32 \mathrm{molH}_{2} \mathrm{O} / \mathrm{m}^{2} / \mathrm{s}\right)$ and the cool $\left(0.21 \mathrm{molH}_{2} \mathrm{O} / \mathrm{m}^{2} / \mathrm{s}\right)$ seasons (Table 2). In any season Gs among the four genotypes were not significantly different.

Diurnal responses of $\operatorname{Tr}$ (Figure 5M-R) followed similar patterns as those of Pn (Figure 5A-F), ETR (Figure 4G-L) and NPQ (Figure 4M-R). Cassava leaves displayed the highest mean Tr across genotypes $\left(6.58 \mathrm{mmolH}_{2} \mathrm{O} / \mathrm{m}^{2} / \mathrm{s}\right)$ in the hot season which was significantly higher $(p<0.001)$ than that in the cool $\left(4.65 \mathrm{mmolH}_{2} \mathrm{O} / \mathrm{m}^{2} / \mathrm{s}\right)$ and rainy $\left(4.52 \mathrm{mmolH}_{2} \mathrm{O} / \mathrm{m}^{2} / \mathrm{s}\right)$ seasons (Table 2). Significant differences in mean Tr among genotypes were detected only in the hot season, with RY11 showing significantly higher mean $(p=0.014)$ than CMR38-125-77.

Diurnal pattern of changes in $\mathrm{Ci} / \mathrm{Ca}$ was depicted in Figure 6A-F showing that $\mathrm{Ci} / \mathrm{Ca}$ was high in the early morning, declining to reach minimum values mostly at 12.30 , then slowly increased in the afternoon. Means of diurnal $\mathrm{Ci} / \mathrm{Ca}$ across genotypes were similar in the cool and hot seasons $(0.70$ and 0.71 , respectively) which were significantly lower $(p<0.001)$ than that in the rainy season $(0.85)$ (Table 2). However, in any season, mean $\mathrm{Ci} / \mathrm{Ca}$ among genotypes did not differ significantly. It was apparent that cassava plants utilized light energy for photosynthesis at different efficiencies in different seasons (Figure 6G-L). The means of LUE across genotypes was highest in rainy season (0.030) and significantly higher $(p<0.001)$ than that in the cool $(0.023)$ and hot $(0.019)$ seasons (Table 2$)$. The means of LUE among genotypes were noted in the rainy and hot seasons. In the rainy season, RY9 had lowest LUE (0.023) which was significantly different $(p=0.045)$ from that of RY11 (0.035) and CMR38-125-77 (0.033). In the hot season, LUE of CMR38-125-77 was lowest (0.016) which was significantly lower $(p=0.041)$ than that of RY11 (0.023). In general, WUE tended to be higher in the early morning then declining throughout the day (Figure $6 \mathrm{M}-\mathrm{R}$ ). Cassava leaves expressed highest means of diurnal WUE across genotypes in the $\mathrm{cool}\left(4.52 \mu \mathrm{molCO} 2 / \mathrm{mmolH}_{2} \mathrm{O}\right)$ season which was significantly higher $(p<0.001)$ than that in the rainy and hot season $\left(2.33\right.$ and $2.05 \mu \mathrm{molCO}{ }_{2} / \mathrm{mmolH}_{2} \mathrm{O}$, respectively). In any season, no significance differences in WUE among genotypes were found. 

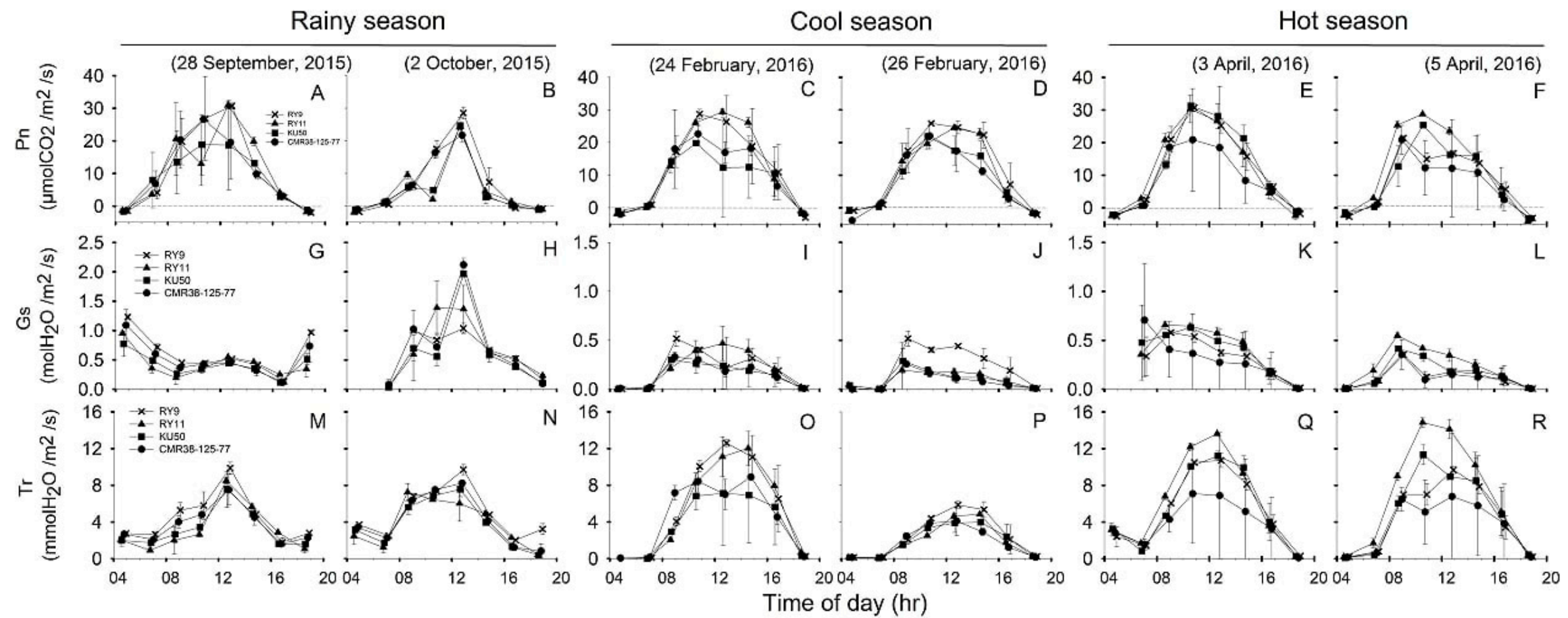

Figure 5. Diurnal pattern of net photosynthetic rate (Pn) (A-F), stomata conductance (Gs) (G-L) and transpiration rate (Tr) (M-R) of four cassava genotypes (X, RY9; $\mathbf{\Delta}$, RY11; $\mathbf{\square}$, KU50 and $\mathbf{0}$, CMR38-125-77) measured from 4:30 to 18:30 at 2-h intervals on two sunny days in rainy (A,B,G,H,M,N), cool (C,D,I,J,O,P), and hot $(\mathbf{E}, \mathbf{F}, \mathbf{K}, \mathbf{L}, \mathbf{Q}, \mathbf{R})$ season. Data shows mean of two replicates \pm SD. 


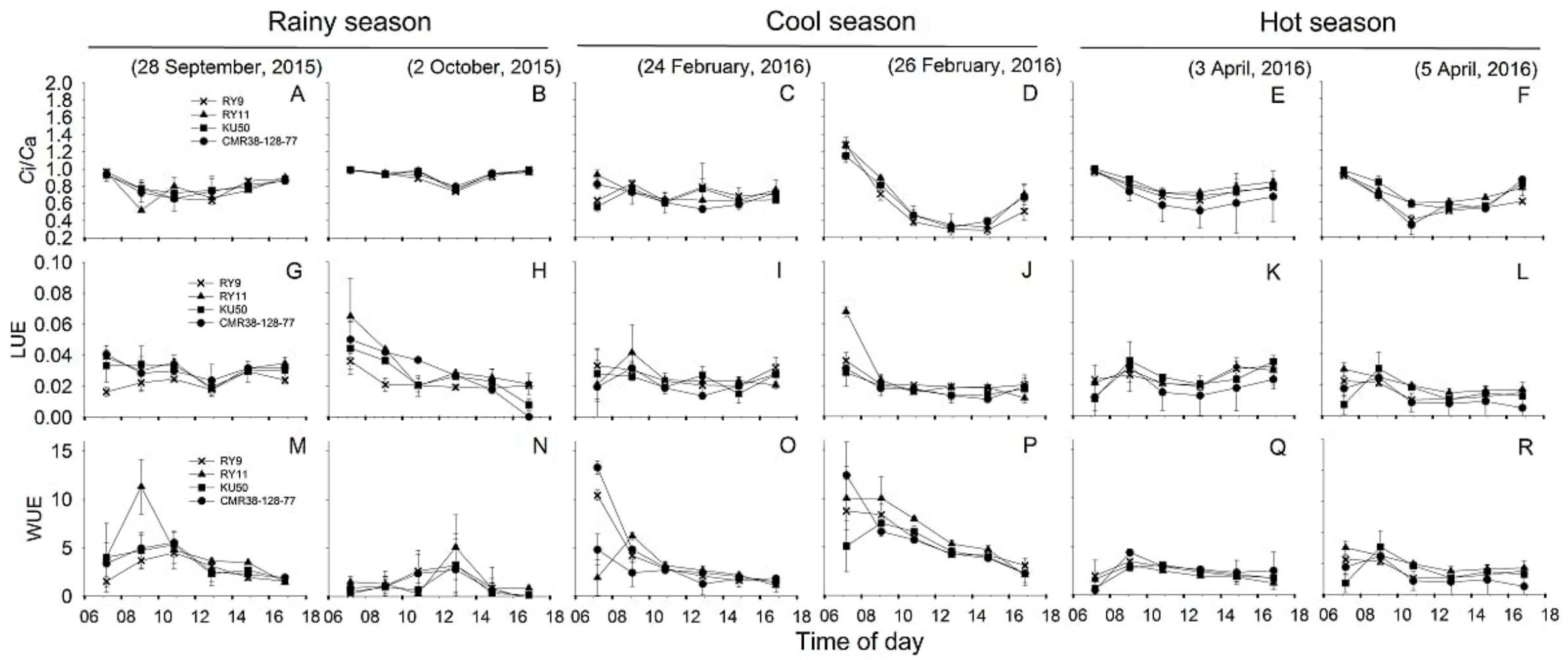

Figure 6. Diurnal pattern of ratio between internal $\mathrm{CO}_{2}$ concentration and ambient $\mathrm{CO}_{2}(\mathrm{Ci} / \mathrm{Ca})(\mathbf{A}-\mathbf{F})$, instantaneous light use efficiency (LUE) (G-L) and instantaneous water use efficiency (WUE) (M-R) of four cassava cultivars (X, RY9; $\mathbf{A}$, RY11; , KU50 and $\mathbf{O}$, CMR38-125-77) measured on two sunny days in rainy (A,B,G,H,M,N), $\operatorname{cool}(\mathbf{C}, \mathbf{D}, \mathbf{I}, \mathbf{J}, \mathbf{O}, \mathbf{P})$, and hot $(\mathbf{E}, \mathbf{F}, \mathbf{K}, \mathbf{L}, \mathbf{Q}, \mathbf{R})$ season. 


\subsection{The Relationship between Cassava Leaf Photosynthesis and Environmental Field Conditions}

The relationships between leaf gas exchange and environmental parameters are demonstrated by the matrix of correlation coefficient values $(r)$ for each season in Table 3. Among the four environmental parameters, $\mathrm{PAR}_{\text {leaf }}$ showed the highest positive correlations with Pn across all seasons. The correlation was highest in the rainy $(r=0.93, p<0.01)$ followed by the cool $(r=0.82, p<0.01)$ and the hot $(r=0.73$, $p<0.01)$ seasons. $\mathrm{T}_{\text {leaf }}$ also expressed highly significant correlations with Pn i.e., $0.76,0.48$ and 0.46 in the rainy, cool and hot seasons, respectively. $\mathrm{RH}_{\mathrm{C}}$, on the other hand, showed highly significant negative correlations with $\mathrm{Pn}$ in all seasons. Pn had significant positive correlations with $\mathrm{VPD}_{\mathrm{L}}$ only in the rainy $(r=0.57, p<0.01)$ and cool $(r=0.33, p<0.01)$ seasons. The relationships between $\operatorname{Tr}$ and all four environmental parameters were highly significant and occurred in the same ways as those for Pn. It is worth noted that the relationships between $\mathrm{Tr}$ and $\mathrm{PAR}_{\text {leaf }}$ had lower correlations than between $\mathrm{Pn}$ and $\mathrm{PAR}_{\text {leaf }}$ but the opposite occurred for $\mathrm{RH}_{\mathrm{C}}$. The relationships between $\mathrm{Ci}$ and all four environmental parameters were also highly significant but occurred in the opposite directions as compared to those for Pn and Tr. The absolute values of correlation coefficient were highest between $\mathrm{Ci}$ and $\mathrm{T}_{\text {leaf }}$ especially in the rainy and hot seasons $(r=-0.90, p<0.01)$. The relationships between $\mathrm{Pn}$ and $\operatorname{Tr}$ were positively correlated in all seasons being higher in the hot $(r=0.75, p<0.01)$ and cool $(r=0.74, p<0.01)$ and lower in the rainy season $(r=0.67, p<0.01)$. The positive correlations between Pn and Gs were highly significant only during the cool and hot seasons, whereas those between $\operatorname{Tr}$ and Gs were highly significant in all seasons i.e., $r=0.73,0.45$ and 0.39 in the cool, hot and rainy season, respectively. Negative correlations between $\mathrm{Pn}$ and $\mathrm{Ci}$ were highest in the rainy season $(r=-0.84$, $p<0.01)$ followed by the cool $(r=-0.63, p<0.01)$ and the hot $(r=-0.50, p<0.01)$ seasons.

Table 3. A correlation matrix of net photosynthetic rate (Pn), stomata conductance (Gs), transpiration rate $(\mathrm{Tr})$, intercellular $\mathrm{CO}_{2}$ concentration $(\mathrm{Ci})$, photosynthetically active radiation $\left(\mathrm{PAR}_{\text {leaf }}\right)$, leaf temperature $\left(\mathrm{T}_{\text {leaf }}\right)$, leaf vapor pressure deficit $\left(\mathrm{VPD}_{\mathrm{L}}\right)$ and canopy relative humidity $\left(\mathrm{RH}_{\mathrm{C}}\right)$. The correlations which are significantly different $(p<0.05$ and $p<0.01)$ are denoted with * and **.

\begin{tabular}{|c|c|c|c|c|c|c|c|c|}
\hline Parameter & Season & Pn & Gs & $\operatorname{Tr}$ & $\mathrm{Ci}$ & PAR $_{\text {leaf }}$ & $\mathrm{T}_{\text {leaf }}$ & $\mathbf{R H}_{\mathbf{C}}$ \\
\hline \multirow[t]{3}{*}{ Gs } & Rainy & $0.59 * *$ & & & & & & \\
\hline & Cool & $0.74^{* *}$ & & & & & & \\
\hline & Hot & $0.50 * *$ & & & & & & \\
\hline \multirow[t]{3}{*}{$\operatorname{Tr}$} & Rainy & $0.67 * *$ & $0.39 * *$ & & & & & \\
\hline & Cool & $0.74^{* *}$ & $0.73^{* *}$ & & & & & \\
\hline & Hot & $0.75^{* *}$ & $0.45^{* *}$ & & & & & \\
\hline \multirow[t]{3}{*}{$\mathrm{Ci}$} & Rainy & $-0.84^{* *}$ & $0.27^{* *}$ & $-0.50 * *$ & & & & \\
\hline & Cool & $-0.63^{* *}$ & -0.21 & $-0.37^{* *}$ & & & & \\
\hline & Hot & $-0.50 * *$ & 0.18 & $-0.54^{* *}$ & & & & \\
\hline \multirow[t]{3}{*}{$\mathrm{PAR}_{\text {leaf }}$} & Rainy & $0.93^{* *}$ & -0.01 & $0.69 * *$ & $-0.79 * *$ & & & \\
\hline & Cool & 0.82 ** & $0.53^{* *}$ & $0.62 * *$ & $-0.67^{* *}$ & & & \\
\hline & Hot & $0.73^{* *}$ & 0.18 & $0.77^{* *}$ & $-0.79 * *$ & & & \\
\hline \multirow{3}{*}{$\mathrm{T}_{\text {leaf }}$} & Rainy & $0.76^{* *}$ & $-0.34^{* *}$ & $0.49 * *$ & $-0.90^{* *}$ & 0.80 ** & & \\
\hline & Cool & $0.48^{* *}$ & $0.28^{* *}$ & $0.66^{* *}$ & $-0.74^{* *}$ & $0.58^{* *}$ & & \\
\hline & Hot & $0.46^{* *}$ & -0.22 & $0.58^{* *}$ & $-0.90^{* *}$ & $0.76^{* *}$ & & \\
\hline \multirow[t]{3}{*}{$\mathrm{RH}_{\mathrm{C}}$} & Rainy & $-0.30^{* *}$ & $-0.19^{* *}$ & $-0.76^{* *}$ & $0.37^{* *}$ & $-0.31^{* *}$ & $-0.34^{* *}$ & \\
\hline & Cool & $-0.54^{* *}$ & $-0.32^{* *}$ & $-0.69 * *$ & $0.69 * *$ & $-0.61^{* *}$ & $-0.93^{* *}$ & \\
\hline & Hot & $-0.50 * *$ & -0.13 & $-0.56^{* *}$ & $0.63 * *$ & $-0.48^{* *}$ & $-0.71^{* *}$ & \\
\hline \multirow[t]{3}{*}{$\mathrm{VPD}_{\mathrm{L}}$} & Rainy & $0.57^{* *}$ & $-0.35^{* *}$ & $0.53^{* *}$ & $-0.78^{* *}$ & $0.65^{* *}$ & $0.86^{* *}$ & $-0.63^{* *}$ \\
\hline & Cool & $0.33^{* *}$ & 0.04 & $0.48^{* *}$ & $-0.61^{* *}$ & $0.43^{* *}$ & $0.85 * *$ & $-0.83^{* *}$ \\
\hline & Hot & 0.16 & $-0.50 * *$ & $0.26^{* *}$ & $-0.79 * *$ & $0.57^{* *}$ & $0.91 * *$ & $-0.44^{* *}$ \\
\hline
\end{tabular}

\section{Discussion}

The results reported here clearly demonstrated cassava resiliency under variable climatic conditions, a trait of great importance for adaptation of the crop to future climate change/global 
warming in the tropics/subtropics where other staple crops might fail [32,33]. The seasonal climatic pattern at the study site varied considerably during the study period. The monthly mean temperature fluctuated from $24{ }^{\circ} \mathrm{C}$ in February 2016 to $32{ }^{\circ} \mathrm{C}$ in April 2016, RH from 80\% in October 2015 to $40 \%$ in April 2016 and rainfall from 323 mm in August 2015 to zero in December 2015 and February 2016 (Figure 1). However, diurnal patterns of photosynthesis (leaf Pn) of field-grown cassava under full irrigation (Figure 5A-F) displayed similar response pattern in different seasons which more or less paralleled with diurnal changes in $\mathrm{PAR}_{\mathrm{A}}$ (Figure 3A-F), despite differences in air temperature and RH. In the rainy season, the daily peaks of Pn occurred at 10:30 or 12:30, depending on genotypes, showing maximum Pn between 27.50 and $36.0 \mu \mathrm{molCO} / 2 / \mathrm{m}^{2} / \mathrm{s}$ (Table 2). Much higher temperature and VPD ${ }_{\text {air }}$ in the hot season could be the major causes of the shift in daily peak of Pn to 8:30 or 10:30 (Figure 5E,F) depending on genotypes while maximum Pn remained high between 31.57 and $33.31 \mu \mathrm{molCO} / 2 / \mathrm{m}^{2} / \mathrm{s}$ (Table 2). Rosenthal et al. [34] measured diurnal Pn of field-grown cassava in Illinois, USA during mild summer (June-August), where total rainfalls were $377 \mathrm{~mm}$, mean daily minimum and maximum temperatures were 18 and $30{ }^{\circ} \mathrm{C}$, and found that these cassava plants also exhibited the Pn peaks around noon with Pn values varying from $18-28 \mu \mathrm{molCO} / 2 / \mathrm{m}^{2} / \mathrm{s}$. In contrast, in a seasonally dry environment, cassava had maximum Pn of $33 \mu \mathrm{molCO} / 2 \mathrm{~m}^{2} / \mathrm{s}$ (average of 10 cultivars) in the early morning (08:00) and decreased thereafter [16]. Therefore, the diurnal patterns of Pn and the range of maximum Pn values in the current study were in accordance with earlier reports.

Variations in diurnal photosynthesis were apparent among genotypes in the cool and hot season. In general, the pre-noon environments were favorable for all four genotypes with small variations in Pn (Figure 5A-F). Nevertheless, during the afternoon Pn of KU50 and CMR38-125-77 tended to be slightly lower than the others particularly in the cool season, while afternoon Pn of CMR38-125-77 had a tendency to be lower than the others in both cool and hot seasons (Figure 5C-F). This could partly be due to lower light incidence on the leaf surface of KU50 and CMR38-125-77 as a result of their prominent leaf-drooping behavior under high light intensity compared to RY9 and RY11 which hardly showed any drooping (see Supplementary Figure S1). Leaf movement in cassava is known as a stress avoidance mechanism in both well-watered and stressed plants $[35,36]$. Genotypic variation in seasonal Pn of field-grown cassava was well-documented in earlier reports [37-39]. A recent study in four African cultivars of cassava (two landraces and two improved lines) under a greenhouse condition showed almost perfect bell-shaped diurnal response with Pn peak at 12:30 and maximum Pn varying from 22 to $27 \mu \mathrm{molCO}_{2} / \mathrm{m}^{2} / \mathrm{s}$ [40].

The relationships between physical parameters of leaf including $\mathrm{PAR}_{\text {leaf, }} \mathrm{T}_{\text {leaf }}, \mathrm{RH}_{\mathrm{C}}$, and $\mathrm{VPD}_{\mathrm{L}}$ and photosynthetic parameters (Pn, Gs, $\mathrm{Tr}$, and $\mathrm{Ci}$ ) in different seasons are expressed in a correlation matrix shown in Table 3. In each season, Pn had the strongest positive correlations with $\mathrm{PAR}_{\text {leaf }}$ (Table 3). Among ecological factors, PAR together with temperature and VPD have been shown to be highly correlated with Pn [41,42]. Diurnal variation in PAR differed in different seasons, and Pn showed a bell-shaped response parallel with PAR during the mild spring while Pn peaked very early in the morning during hot summer [40]. In this study, although maximum $\mathrm{PAR}_{\mathrm{A}}$ and $\mathrm{PAR}_{\text {leaf }}$ occurred in the rainy followed by the hot and the cool season (Table 1 ), the mean daily PAR $_{\text {leaf }}$ values were lowest in the rainy season $(p<0.001$, Table 1$)$ due to frequent cloud cover causing highly fluctuating $\mathrm{PAR}_{\mathrm{A}}$ (Figure $\left.3 \mathrm{~A}, \mathrm{~B}\right)$. Lowest mean daily $\mathrm{PAR}_{\text {leaf }}$ in the rainy season may attribute to lower mean daily Pn $\left(11.75 \mu \mathrm{molCO}_{2} / \mathrm{m}^{2} / \mathrm{s}, p=0.044\right)$ than the cool and hot seasons (Table 2). A recent report [43] also pointed out that solar radiation is a limiting factor in rainy season based on comparison between maximum net photosynthetic rates (Pnmax) from light response curve and the predicted Pn from actual solar radiation data. It is known that Pn in fully expanded young cassava leaves developed under sunny warm climate does not reach light saturation even up to $1800 \mu \mathrm{mol} / \mathrm{m}^{2} / \mathrm{s}$ [41]. In each season, Pn were significantly correlated with PAR leaf as well as $\mathrm{T}_{\text {leaf }}, \mathrm{RH}_{\mathrm{C}}$ and $\mathrm{VPD}_{\mathrm{L}}$ (Table 3). In spite of the significant differences $(p<0.001)$ in mean $\mathrm{T}_{\text {air }}$ and $\mathrm{VPD}_{\text {air }}$ between the cool and hot seasons (Table 1), well-watered cassava in this study performed equally well showing the daily mean Pn of 14.60 and $14.32 \mu \mathrm{molCO} 2 / \mathrm{m}^{2} / \mathrm{s}$, respectively (Table 2). Moreover, it is worth noting that in the 
hot season cassava genotype RY11 could maintain maximum daily Pn at $28.9 \mu \mathrm{molCO} / \mathrm{m}^{2} / \mathrm{s}$ while leaf temperature reached $39.8^{\circ} \mathrm{C}$. Previous studies reported optimum temperature range for cassava photosynthesis in tropical environments between $30-35^{\circ} \mathrm{C}[38,44]$. This indicated that these improved cassava genotypes have been well-adapted to environments in different seasons in this climatic zone. Cassava can be widely adapted to environments and usually requires a warm climate with high solar radiation for optimum photosynthesis, growth and productivity [44].

Among leaf gas exchange parameters, in the rainy season under fluctuating light intensity but high $\mathrm{RH}$, higher correlation was found between Pn and $\mathrm{Ci}(-0.84, p<0.01)$ than between Pn and Gs $(0.59$, $p<0.01$ ) (Table 3) indicating a stronger role of photosynthetic capacity on $\mathrm{CO}_{2}$ fixation or non-stomatal regulation as compared to stomatal control of photosynthesis. Gas exchange measurements of 15 cassava cultivars mostly during high rainfall periods also found higher correlation between Pn and $\mathrm{Ci}(-0.84)$ than Pn and Gs (0.40) [39]. Non-stomatal limitation may be attributed to mesophyll resistance to $\mathrm{CO}_{2}$ flux, carboxylation efficiency of Rubisco and RuBP regeneration [45]. The extent to which Rubisco limits photosynthesis depends largely on irradiance [46]. Therefore, low mean daily Pn in the rainy season could be attributed to low activity of Rubisco under fluctuating and low mean daily PAR (Figure 3A,B; Table 1). In the cool and hot seasons, Pn was influenced by both stomatal and non-stomatal controls as indicated by highly significant correlations $(p<0.01)$ between Pn and Gs, and Pn and Ci (Table 3). In the cool season, higher correlation between Pn and Gs (0.74, $p<0.01)$ than Pn and $\mathrm{Ci}(0.63, p<0.01)$ may indicate stronger role of stomatal limitation inferred by lowest $\mathrm{Gs}\left(0.21 \mathrm{molH}_{2} \mathrm{O} / \mathrm{m}^{2} / \mathrm{s} ; p<0.001\right)$ in the cool season (Table 3). In the cool and hot season, $\mathrm{RH}_{\text {air }}$ was significantly lower $(p<0.001)$ than that in the rainy season (Table 1$)$. Cassava is very sensitive to low air humidity and its stomatal conductance rapidly decreases in response to dry air irrespective of soil water conditions [44,47]. In the cool and hot season, maximum Gs of approximately $0.5 \mathrm{molH}_{2} \mathrm{O} / \mathrm{m}^{2} / \mathrm{s}$ occurred at 08:30 or 10:30 (Figure $5 \mathrm{I}-\mathrm{L}$ ) coinciding with high $\mathrm{RH}$ and low $\mathrm{VPD}_{\mathrm{L}}$ in the early morning (Figure 3O-R,U-X), thereafter Gs declined in parallel with decreasing RH and increasing $\mathrm{VPD}_{\mathrm{L}}$ resulting in decreasing Pn during the afternoon. Nevertheless, under irrigation, cassava was able to maintain relatively high mean daily $\operatorname{Pn}\left(14.60\right.$ and $14.32 \mu \mathrm{molCO} / 2 / \mathrm{m}^{2} / \mathrm{s}$; Table 2) while mean Gs values were higher than $0.15 \mathrm{molH}_{2} \mathrm{O} / \mathrm{m}^{2} / \mathrm{s}$ (Table 2) which is the threshold value above which the plants would be considered under non- or mild water stress conditions [48]. It has been suggested that photosynthesis metabolism is substantially resistant to water stress until Gs is below $0.1-0.15 \mathrm{molH}_{2} \mathrm{O} / \mathrm{m}^{2} / \mathrm{s}$ [49].

Diurnal changes in transpiration rates (Tr) paralleled closely those of Pn (Figure 3A-F,M-R) and had highly significant correlations with Gs (Table 3). This indicated that transpiration was greatly influenced by stomatal regulation particularly in the hot season. Transpiration rates were similar in the rainy and cool seasons $\left(4.52\right.$ and $\left.4.65 \mathrm{mmolH}_{2} \mathrm{O} / \mathrm{m}^{2} / \mathrm{s}\right)$, and significantly higher $(p<0.001)$ in the hot $\left(6.58 \mathrm{mmolH}_{2} \mathrm{O} / \mathrm{m}^{2} / \mathrm{s}\right)$ season (Figure $5 \mathrm{M}-\mathrm{R}$, Table 2) coinciding with increasing $\mathrm{VPD}_{\text {air }}$ and $\mathrm{VPD}_{\mathrm{L}}$ in the latter (Figure $3 \mathrm{~S}-\mathrm{X}$, Table 1). Pronounced effects of $\mathrm{VPD}_{\mathrm{L}}$ on stomatal movement and transpiration rates were classically demonstrated in cassava [47]. Similar seasonal variation was reported in orange trees in which transpiration rates of well-watered orange plants in summer were about 2.5 folds higher than that in winter [50]. Highest $\mathrm{Tr}$ in the hot season resulted in lowest WUE $\left(2.05 \mu \mathrm{molCO}_{2} / \mathrm{mmol} \mathrm{H}_{2} \mathrm{O}\right)$ in the hot which was significantly lower $(p<0.001)$ than that in the cool season (Table 2). High WUE in the cool season $\left(4.52 \mu \mathrm{molCO} / 2 / \mathrm{mmol} \mathrm{H}_{2} \mathrm{O}\right)$ was due to lower $\mathrm{Tr}$ $\left(4.65 \mathrm{mmol} / \mathrm{m}^{2} / \mathrm{s}\right)$ as a result of partially closed stomata in response to dry air (Table 2). El-Sharkawy and De Tafur [39] reported similar WUE value of $4.5 \mu \mathrm{molCO} / 2 / \mathrm{mmol} \mathrm{H}_{2} \mathrm{O}$ which was averaged from numerous measurements performed from upper canopy leaves of 15 cassava cultivars. Mean Tr among genotypes were significantly different $(p=0.014)$ only in the hot season i.e., the values appeared in the order RY11 > KU50 = RY9 > CMR38-125-7, the same order as for Gs (Table 2). High Tr played crucial role in heat dissipation, therefore maximum $\mathrm{T}_{\text {leaf }}$ in the hot season was highest in CMR38-125-77 $\left(44.3^{\circ} \mathrm{C}\right)$ and lowest $\left(41.0^{\circ} \mathrm{C}\right)$ in RY11 and KU50 (Table 1). Lower Tr in RY9 and CMR38-125-77 was related to higher WUE, although not statistically significant, than RY11 and KU50 (Table 2). 
When measured under natural rainfed environments, 15 cultivars of cassava exhibited large variations in WUE from 3.89 to $4.74 \mu \mathrm{molCO}_{2} / \mathrm{mmol} \mathrm{H}_{2} \mathrm{O}$ [39].

The observation that maximum quantum yield of PSII photochemistry $\left(\mathrm{F}_{\mathrm{v}} / \mathrm{F}_{\mathrm{m}}\right)$ values of all four cassava genotypes at 04:30 (predawn) were between 0.823-0.878 (Table 2) across all three seasons indicated that cassava leaves were healthy, and no chronic damages occurred in PSII [51]. However, significantly $(p<0.001)$ lower $\mathrm{F}_{\mathrm{v}} / \mathrm{F}_{\mathrm{m}}$ was observed in the cool season compared to the others. Negative effects of low temperature (in winter and spring) on reduction of $F_{v} / F_{m}$ have been reported in roses [52] and temperate bamboo [53]. Energy utilization by a leaf is indicated by diurnal changes in the effective quantum yield of PSII photochemistry $\left(\Phi_{\text {PSII }}\right)$ which changed in the opposite direction as PAR and showed similar patterns in all seasons. However, as shown in Figure 4A-F, the recovery of $\Phi_{\text {PSII }}$ in the afternoon occurred much earlier in rainy season (average $\Phi_{\text {PSII }}$ at 14:30 was 0.77 ) than in the cool and hot seasons (average $\Phi_{\mathrm{PSII}}$ at 14:30 were 0.43 and 0.53 in the cool and hot season, respectively). In addition, mean daily $\Phi_{\mathrm{PSII}}$ across genotypes was significantly higher $(p<0.001)$ than in the hot and cool seasons (Table 2). This indicated the interactive effects between light intensity and other environmental parameters such as temperature and VPD in the cool and hot seasons. Even though the soil moistures were optimized due to irrigation, stressful environments in the cool and hot season clearly posed negative effects on energy utilization [40,54].

The patterns of diurnal changes in ETR (Figure 4G-R) were similar and parallel to the curves of PAR (Figure 3A-F). The $\Phi_{\text {PSII }}$ and the derived ETR are dependent on ambient PAR. Hence, mean daily ETR across genotypes in the rainy season was significantly lower $(p<0.001)$ than the others (Table 2). Theoretically, under controlled conditions $\Phi_{\text {PSII }}$ and ETR are accurately correlated with $\mathrm{Pn}$ and can be used to predict $\mathrm{CO}_{2}$ assimilation and hence productivity [28]. Nevertheless, under stressful environments during the afternoon in the hot season, Pn of RY9 peaked at 08:30 on the 5 April 2016 which was the hotter day (Figure 5F) while ETR continued to increase to reach maximum at 12:30 (Figure 4L). Similar results were observed in cassava cv. RY9 under both irrigated and rainfed conditions [55] and also in other plants under irrigation such as soybean [56] and peach palms [57]. This indicated that after 08:30, under limited $\mathrm{CO}_{2}$ availability due to stomatal closure after 8.30 (Figure 5K,L), higher proportion of reductants generated from electron transport could be allocated to alternative electron sinks most commonly photorespiration, Mehler reactions and cyclic electron flow $[58,59]$. These alternative pathways served to balance photosynthesis electron transfer so that light energy is optimally used for $\mathrm{CO}_{2}$ fixation and over-reduction of electron carriers and excess generation of reactive oxygen species are prevented [60]. The diurnal patterns of NPQ curves were parallel to the curves of PAR because NPQ operated to dissipate excess light energy as heat to protect PSII from photodamage [61,62]. Both PAR and NPQ across genotypes were highest in the hot followed by the cool and significantly lower $(p<0.001)$ in the rainy season (Tables 1 and 2$)$. Moreover, similar to other previously mentioned parameters (Pn, $\Phi_{\mathrm{PSII}}$ and ETR), genotypic variations in NPQ were more apparent in the hot season than the others. Compared with the others CMR38-125-77 had a tendency to have lowest $\Phi_{\mathrm{PSII}}$ and ETR but highest NPQ (Figure 4E,F,K,L,Q,R) during 10:30-14:30 in the hot season, indicating the most active photoprotective mechanisms. The most prominent component of NPQ is qE which harmlessly dissipated excess light energy as heat through functioning of the xanthophyll cycle and PsbS protein [63]. It was suggested that more PsbS protein and hence increasing qE capacity might improve crop production in adverse environments [64]. Despite lowest leaf-level Pn in the hot season (Table 2), CMR-38-125-77 had better growth than the others as evidenced by significantly higher leaf production rate and LAI (Figure 2). A recent report in rice showed that rice transgenic line overexpressing PsbS protein which regulated qE had higher NPQ than wild type but comparable leaf-level ETR and Pn. Higher NPQ in the transgenic rice line efficiently protected PSII from photodamage, hence displayed better growth, higher leaf area per plant, higher photosynthetic performance, greater total biomass and finally higher grain yield than the wild type [65].

Although leaf-level Pn was lowest in the rainy season cassava plants had much better growth, as indicated by significantly higher $(p<0.001)$ means across genotypes of rate of increase in plant height 
and LAI than in the cool and hot seasons (Figure 2; Table S2). In relation to photosynthesis and early vegetative growth (0-3 months), all four genotypes performed equally well in the rainy and cool season (Figure 2). However, in the hot season, CMR-38-125-77 showed significantly higher leaf production rate and LAI than the others (Figure 2). Importantly, at the age of 3 months CMR38-125-77 growing in the hot season had LAI of $3.49 \mathrm{~m}^{2} \mathrm{~m}^{-2}$, not significantly different from those growing in the rainy season $\left(\mathrm{LAI}=3.57 \mathrm{~m}^{2} \mathrm{~m}^{-2}\right)$, whereas hot-season LAI values of the other genotypes showed $20-37 \%$ reduction from those in the rainy season crop (Figure 2, Table S2). Although CMR38-125-77 had a moderate leaf-level Pn, its high WUE as well as efficient protective mechanisms (high NPQ and leaf drooping) were beneficial for its growth performance and canopy development. Since analyses of cassava growth and yield are usually evaluated on the basis of both LAI and Pn [14,66], it can be concluded that among the studied genotypes CMR38-125-77 is most suitable for planting in the post-rainy season (in December) to obtain good vegetative growth during the first 3 months. Cock et al. [9] suggested that cassava plants should reach LAI of $3.0 \mathrm{~m}^{2} \mathrm{~m}^{-2}$ as quickly as possible in order to obtain good root yield. Similar results were obtained in a parallel experiment at the same site that CMR38-125-77 planted in December had highest LAI at 4 months after planting and subsequently gave highest storage root yield [12]. Moreover, Sawatraksa et al. [67], who studied the same set of genotypes planted in December under rain-fed paddy field conditions, found that CMR38-125-77 had the highest biomass during early vegetative growth.

\section{Conclusions}

Growing cassava in the tropical savanna climate under irrigation, the environmental conditions in the rainy season were the most favorable for early vegetative growth of all four cassava genotypes, based on rate of increase in plant height and LAI at 3 MAP. Among the four genotypes, CMR38-125-77 was the most suitable genotype to be planted in December and growing during the period from cool to hot season, based on its highest rate of leaf production and LAI at 3 MAP. Mean daily net photosynthesis and electron transport rates in the rainy season were slightly lower than those in the cool and hot season due to fluctuating light intensity. Cassava plants displayed several morphological and physiological mechanisms in the hot season, to protect photosynthesis machinery from being damaged under the conditions of high light intensity, temperature and VPD, by leaf drooping, early stomatal closure, enhanced transpiration, thermal dissipation by NPQ and diversion of electrons to alternative sinks, and different genotypes may employ different strategies to varying extent.

Supplementary Materials: The following are available online at http://www.mdpi.com/2073-4395/9/4/206/s1, Figure S1: leaf drooping at midday of cassava cultivars; RY9 (A), RY11 (B), KU50 (C) and CMR38-125-77 (D) growing in the field under irrigated condition. Table $S 1$ : ambient photosynthetically active radiation $\left(\mathrm{PAR}_{\mathrm{A}}\right)$, air temperature $\left(\mathrm{T}_{\text {air }}\right)$, relative humidity $\left(\mathrm{RH}_{\mathrm{air}}\right)$, air vapor pressure deficit $(\mathrm{VPD}$ air $)$, rainfall and irrigation for the three planting dates (PD). The environmental parameters were recorded from the date of planting until the date when photosynthesis measurements were performed on the three-month-old plants. Environmental parameters for PD-Jun, PD-Nov and PD-Dec were measured during 30 June-27 September 2015, 10 November 2015-23 February 2016 and 15 December 2015-2 April 2016, respectively. The values are daily minima, maxima and means of data collected every $5 \mathrm{~min}$ on each day, and the data were then averaged over days. Lower case letters indicate significance differences $(p<0.05)$ among planting dates. Table S2: the minima, maxima and means of growth parameters including rate of increase in plant height, rate of leaf production and leaf area index (LAI) of three-month-old plants of cassava genotypes RY9, RY11, KU50 and CMR38-125-77 planted in June 2015 (PD-Jun), November 2015 (PD-Nov) and December 2015 (PD-Dec). Means which are significantly different $(p<0.05)$ among seasons are denoted with capital letters. Means which are significantly different $(p<0.05)$ among genotypes are denoted with different lower case letters.

Author Contributions: Conceptualization, S.S., P.T., K.V., P.B., N.V., and S.J.; investigation and data collection, S.S.; data analysis, S.S. and P.T.; methodology, S.S., P.T., K.V., P.B., N.V., and S.J.; supervision, P.T.; writing (original draft preparation), S.S. and P.T.; writing (review and editing), S.S., P.T., K.V., P.B., N.V., S.J. and S.R.

Funding: This project was financially supported by the Thailand Research Organizations Network (TRON) administered by the National Science and Technology Development Agency (NSTDA). The first author is supported by Ph.D. scholarship from the National Science and Technology Development Agency (NSTDA) under the Thailand Graduate Institute of Science and Technology (TGIST), Grant no. TG-44-12-60-009D. The authors 
also acknowledge the Thailand Research Fund (Project code: IRG5780003) and Faculty of Agriculture, Khon Kaen University for providing financial support for manuscript preparation activities.

Acknowledgments: We would like to thank the member of cassava team project and salt-tolerant rice research group at KKU for field and data collection.

Conflicts of Interest: The authors declare no conflict of interest.

\section{Abbreviations}

\begin{tabular}{|c|c|}
\hline APAR & absorbed photosynthetically active radiation \\
\hline $\mathrm{Ca}$ & ambient $\mathrm{CO}_{2}$ concentration \\
\hline $\mathrm{Ci}$ & intercellular $\mathrm{CO}_{2}$ concentration \\
\hline Chl & chlorophyll \\
\hline $\operatorname{Tr}$ & transpiration rate \\
\hline ETR & electron transfer rate \\
\hline $\mathrm{F}_{0}$ & minimal fluorescence yield of the dark-adapted state \\
\hline $\mathrm{F}^{\prime}$ & steady state fluorescence in the light-adapted state \\
\hline$F_{m}$ & the maximal fluorescence of the dark-adapted state \\
\hline $\mathrm{F}_{\mathrm{m}}^{\prime}$ & the maximal fluorescence of the light-adapted state \\
\hline $\mathrm{F}_{\mathrm{v}} / \mathrm{F}_{\mathrm{m}}$ & the maximal photochemical quantum yield of PSII \\
\hline Gs & stomatal conductance \\
\hline LUE & light-use efficiency (=Pn/APAR) \\
\hline LAI & leaf area index \\
\hline NPQ & nonphotochemical quenching \\
\hline Pn & net photosynthetic rate \\
\hline$\Phi_{\text {PSII }}$ & effective quantum yield of PSII photochemistry \\
\hline PAR & photosynthetically active radiation \\
\hline $\mathrm{PAR}_{\text {leaf }}$ & photosynthetically active radiation on the leaf surface \\
\hline $\mathrm{PAR}_{\mathrm{A}}$ & ambient photosynthetically active radiation \\
\hline PSII & photosystem II \\
\hline$r$ & correlation coefficient \\
\hline $\mathrm{R}$ & respiration rate \\
\hline $\mathrm{RH}_{\text {air }}$ & air relative humidity \\
\hline $\mathrm{RH}_{\mathrm{C}}$ & canopy relative humidity \\
\hline $\mathrm{T}_{\text {air }}$ & air temperature \\
\hline $\mathrm{T}_{\text {leaf }}$ & leaf temperature \\
\hline $\mathrm{VPD}_{\mathrm{A}}$ & air vapor pressure deficit \\
\hline $\mathrm{VPD}_{\mathrm{L}}$ & leaf-to-air vapor pressure deficit \\
\hline WUE & water-use efficiency $(=\mathrm{Pn} / \mathrm{Tr})$ \\
\hline
\end{tabular}

\section{References}

1. Burns, A.; Gleadow, R.; Cliff, J.; Zacarias, A.; Cavagnaro, T. Cassava: The drought, war and famine crop in a changing world. Sustainability 2010, 2, 3572-3670. [CrossRef]

2. Rosenthal, D.M.; Ort, D.R. Examining cassava's potential to enhance food security under climate change. Trop Plant Biol. 2011, 4, 30-38. [CrossRef]

3. Tonukari, N.; Ezedom, T.; Enuma, C.C.; Sakpa, S.O.; Avwioroko, O.J.; Eraga, L.; Odiyoma, E. White gold: Cassava as an industrial base. Am. J. Plant Sci. 2015, 6, 972-979. [CrossRef]

4. Food and Agriculture Organization Corporate Statistical Database. Crops. Available online: http://www.fao. org/faostat/en/\#data/QC/visualize (accessed on 7 April 2019).

5. Ministry of Agriculture and Cooperative, Thailand. Status of Cassava in Thailand: Implications for Future Research and Development. Available online: http://www.fao.org/3/y1177e/Y1177E04.htm (accessed on 24 January 2019).

6. Thailand Ministry of Agriculture and Cooperative. Good Agricultural Practices for Cassava; National Bureau of Agricultural Commodity and Food Standards Ministry of Agriculture and Cooperatives: Bangkok, Thailand, 2008. 
7. Hammer, G.L.; Hobman, F.R.; Shepherd, R.K. Effects of planting time and harvest age on cassava (Manihot esculenta) in northern Australia. I. Crop growth and yield in moist environments. Exp. Agric. 1987, 23, 401-414. [CrossRef]

8. El-Sharkawy, M.A. Prospects of photosynthetic research for increasing agricultural productivity, with emphasis on the tropical $C_{4}$ Amaranthus and the cassava $C_{3}-C_{4}$ crops. Photosynthetica 2016, 54, 161-184. [CrossRef]

9. Cock, J.H.; Franklin, D.; Sandoval, G.; Juri, P. The ideal cassava planting for maximum yield. Crop Sci. 1979, 19, 271-279. [CrossRef]

10. El-Sharkawy, M.A. Effect of humidity and wind on leaf conductance of field growth cassava. Rev. Bras. Fisiol. Veg. 1990, 2, 17-22.

11. Office of Agricultural Economics. Agricultural Production Index. Available online: http://www.oae.go.th/ view/1/Home/EN-US (accessed on 23 January 2019).

12. Phoncharoen, P.; Banterng, P.; Vorasoot, N.; Jogloy, S.; Theerakulpisut, P.; Hoogenboom, G. Growth rates and yields of cassava at different planting dates in a tropical savanna climate. Sci. Agric. 2018, in press.

13. Furbank, R.T.; Quick, W.P.; Sirault, X.R.R. Improving photosynthesis and yield potential in cereal crops by targeted genetic manipulation: Prospects, progress and challenges. Field Crops Res. 2015, 182, 19-29. [CrossRef]

14. De Souza, A.P.; Massenburg, L.N.; Jaiswal, D.; Jaiswal, D.L.; Cheng, S.L.; Shekar, R.L.; Long, S.P. Rooting for cassava: Insights into photosynthesis and associated physiology as photosynthesis and associated physiology as a route to improve yield potential. New Phytol. 2016, 213, 50-65. [CrossRef] [PubMed]

15. El-Sharkawy, A.M.; Lopez, Y.; Bernal, L.M. Genotypic variations in activities of phosphoenolpyruvate carboxylase and correlations with leaf photosynthetic characteristics and crop productivity of cassava grown in low-land seasonally-dry tropics. Photosynthetica 2008, 46, 238-247. [CrossRef]

16. De Tafur, S.M.; El-Sharkawy, A.M.; Calle, F. Photosynthesis and yield performance of cassava in seasonally dry and semiarid environments. Photosynthetica 1997, 33, 249-257. [CrossRef]

17. Baker, N.R.; Rosenqvist, E. Applications of chlorophyll fluorescence can improve crop production strategies: An examination of future possibilities. J. Exp. Bot. 2004, 403, 1607-1621. [CrossRef] [PubMed]

18. Brestic, M.; Zivcak, M.; Kunderlikova, K.; Allakhverdiev, S.I. High temperature specifically affects the photoprotective responses of chlorophyll b-deficient wheat mutant lines. Photosynth. Res. 2016, 130, 251-266. [CrossRef]

19. Loomis, R.S.; Rabbinge, R.; Ng, E. Explanatory models in crop physiology. Annu. Rev. Plant Physiol. 1979, 30, 339-367. [CrossRef]

20. Polthanee, A.; Janthajam, C.; Promkhambut, A. Growth, yield and starch content of cassava following rainfed lowland rice in Northeast Thailand. Int. J. Environ. Res. 2014, 9, 319-324.

21. Kottek, M.; Grieser, J.; Beck, C.; Rudolf, B.; Rubel, F. World map of the Köppen-Geiger climate classification updated. Meteorol. Z. 2006, 5, 259-263. [CrossRef]

22. Thai Meteorological Department. Climate of Thailand Report. Available online: https://www.tmd.go.th/en/ archive/thailand_climate.pdf (accessed on 11 September 2018).

23. Howeler, R.H. Cassava mineral nutrition and fertilization. In Cassava: Biology, Production and Utilization; Hillocks, R.J., Thresh, J.M., Bellotti, A.C., Eds.; CABI Publishing: New York, NY, USA, 2002; pp. 149-166.

24. Phoncharoen, P.; Banterng, P.; Vorasoot, N.; Jogloy, S.; Theerakulpisut, P.; Hoogenboom, G. The impact of seasonal environments in a tropical savanna climate on forking, leaf area index, and biomass of cassava genotypes. Agron. J. 2019, 9, 19. [CrossRef]

25. Welles, J.M.; Cohen, S. Canopy structure measurement by gap fraction analysis using commercial instrumentation. J. Exp. Bot. 1996, 47, 1335-1342. [CrossRef]

26. Alves, A.A.C. Cassava Botany and Physiology. In Cassava: Biology, Production and Utilization; Hillock, R.J., Thres, J.M., Bellotti, A.C., Eds.; CABI Publishing: New York, NY, USA, 2002; pp. 67-89.

27. Kalaji, H.M.; Schansker, G.; Ladle, R.J.; Goltsev, V.; Bosa, K.; Allakhverdiev, S.I.; Brestic, M.; Bussotti, F.; Calatayud, A.; Dabrowski, P.; et al. Frequently asked questions about in vivo chlorophyll fluorescence: Practical issues. Photosynth. Res. 2014, 122, 121-158. [CrossRef]

28. Murchie, E.H.; Lawson, T. Chlorophyll fluorescence analysis: A guide to good practice and understanding some new applications. J. Exp. Bot. 2013, 64, 3983-3998. [CrossRef]

29. Sigmaplot Version 11.0; Systat Software, Inc.: San Jose, CA, USA, 1996. 
30. MSTAT-C Version 1.42; Michigan State University: East Lansing, MI, USA, 1992.

31. Gomez, K.A.; Gomez, A.A. Statistical Procedures for Agricultural Research; John Wiley and Sons: New York, NY, USA, 1984.

32. Jarvis, A.; Ramirez-Villegas, J.; Herrera Campo, J.B.; Navarro-Racines, C. Is cassava the answer to African climate change adaptation? Trop. Plant Biol. 2012, 51, 9-29. [CrossRef]

33. El-Sharkawy, M.A. Global warming: Causes and impacts on agroecosystems productivity and food security with emphasis on cassava comparative advantage in the tropics/subtropics. Photosynthetica 2014, 52, 161-178. [CrossRef]

34. Rosenthal, D.M.; Slattery, R.A.; Miller, R.E.; Grennan, A.K.; Cavagnaro, T.R.; Fauquet, C.M.; Gleadow, R.M.; Ort, D.R. Cassava about-FACE: Greater than expected yield stimulation of cassava (Manihot esculenta) by future $\mathrm{CO}_{2}$ levels. Glob. Chang. Biol. 2012, 18, 2661-2675. [CrossRef]

35. El-Sharkawy, M.A. Cassava biology and physiology. Plant Mol. Biol. 2004, 56, 481-501. [CrossRef] [PubMed]

36. Rosa, L.M.; Forseth, I.N. Diurnal patterns of soybean leaf inclination angles and azimuthal orientation under different levels of ultraviolet-B radiation. Agric. For. Meteorol. 1995, 78, 107-119. [CrossRef]

37. El-Sharkawy, M.A. Utility of basic research in plant/crop physiology in relation to crop improvement: A review and a personal account. Braz. J. Plant Physiol. 2007, 18, 419-446. [CrossRef]

38. El-Sharkawy, M.A. Physiological characteristics of cassava tolerance to prolonged drought in the tropics: Implication for breeding cultivars adapted to seasonally dry and semiarid environments. Braz. J. Plant Physiol. 2007, 19, 257-286. [CrossRef]

39. El-Sharkawy, M.A.; De Tafur, S.M. Genotypic and within canopy variation in leaf carbon isotope discrimination and its relation to short-term leaf gas exchange characteristics in cassava grown under rain-fed conditions in the tropics. Photosynthetica 2007, 45, 515-526. [CrossRef]

40. De Souza, A.P.; Long, S.P. Toward improving photosynthesis in cassava: Characterizing photosynthetic limitations in four current African cultivars. Food Energy Secur. 2018, 7, 1-14. [CrossRef]

41. El-Sharkawy, M.A. International research on cassava photosynthesis, productivity, eco-physiology, and responses to environmental stresses in the tropics. Photosynthetica 2006, 44, 481-512. [CrossRef]

42. Song, Y.; Chen, Q.; Ci, D.; Shao, X.; Zhang, D. Effects of high temperature on photosynthesis and related gene expression in poplar. BMC Plant Biol. 2014, 14. [CrossRef] [PubMed]

43. Vongchareon, K.; Santanoo, S.; Banterng, P.; Jogloy, S.; Vorasoot, N.; Theerakulpisut, P. Seasonal variation in photosynthesis performance of cassava at two different growth stages under irrigated and rain-fed conditions in a tropical savanna climate. Photosynthetica 2018, 56, 1398-1413. [CrossRef]

44. El-Sharkawy, M.A. Stress-tolerant cassava: The role of integrative ecophysiology-breeding research in crop improvement. OJSS 2012, 2, 162-186. [CrossRef]

45. Grassi, G.; Magnani, F. Stomatal, mesophyll conductance and biochemical limitations to photosynthesis as affected by drought and leaf ontogeny in ash and oak trees. Plant Cell Environ. 2005, 28, 834-849. [CrossRef]

46. Stitt, M.; Schulze, D. Does Rubisco control the rate of photosynthesis and plant growth-An exercise in molecular ecophysiology. Plant Cell Environ. 1994, 17, 465-487. [CrossRef]

47. El-Sharkawy, M.A.; Cock, J.H. The humidity factor in stomatal control and its effect on crop productivity. In Biological Control of Photosynthesis; Marcelle, R., Clijsters, H., Van Poucke, M., Eds.; Springer: Dordrecht, The Netherlands, 1986; pp. 187-198.

48. Flexas, J.; Medrano, H. Drought inhibition of photosynthesis in $\mathrm{C}_{3}$ plants: Stomatal and non-stomatal limitations revisited. Ann. Bot. 2002, 89, 183-189. [CrossRef] [PubMed]

49. Flexas, J.; Bota, J.; Cifre, J.; Escalona, J.M.; Galmes, J.; Gulias, J.; Lefi, E.; Martinez-Canellas, S.F.; Moreno, M.T.; Ribas-Carbo, M.; et al. Understanding down regulation of photosynthesis under water stress: Future prospects and searching for physiological tools for irrigation management. Ann. Appl. Biol. 2004, 144, 273-283. [CrossRef]

50. Ribeiro, R.V.; Machado, E.C.; Santos, M.G.; Oliveira, R.F. Photosynthesis and water relations of well-watered orange plants as affected by winter and summer conditions. Photosynthetic 2009, 47, 215-222. [CrossRef]

51. Breatic, M.; Zivcak, M. PSII Fluorescence techniques for measurement of drought and hight temperatures stress signal in crop plants: Protocols and application. In Molecular Stress Physiology of Plants; Rout, G.R., Das, A.B., Eds.; Springer: Dordrecht, The Netherlands, 2013; pp. 87-131. 
52. Pompodakis, N.E.; Terry, L.A.; Joyce, D.C.; Lydakis, D.E.; Papadimitriou, M.D. Effect of seasonal variation and storage temperature on leaf chlorophyll fluorescence and vase life of cut roses. Postharvest Biol. Technol. 2005, 36, 1-8. [CrossRef]

53. Van Goethem, D.; De Smedt, S.; Valcke, R.; Potters, G.; Samson, R. Seasonal, diurnal and vertical variation of chlorophyll fluorescence on Phyllostachys humilis in Ireland. PLoS ONE 2013, 8, e72145. [CrossRef]

54. Brestic, M.; Zivcak, M.; Kalaji, M.H.; Carpentier, R.; Allakhverdiev, S.I. Photosystem II themostability in situ: Enviromentally induced acclimation and genotype-specific reaction in Triticum aeativum L. Plant Physiol. Biochem. 2012, 57, 93-105. [CrossRef]

55. Vongchareon, K.; Santanoo, S.; Banterng, P.; Jogloy, S.; Vorasoot, N.; Theerakulpisut, P. Diurnal and seasonal variations in the photosynthetic performance and chlorophyll fluorescence of cassava 'Rayong 9' under irrigated and rainfed conditions. Photosynthetica 2019, 57, 268-285. [CrossRef]

56. Huang, L.F.; Zheng, J.H.; Zhang, Y.Y.; Hu, W.H.; Mao, W.H.; Zhou, Y.H.; Yu, J.Q. Diurnal variations in gas exchange, chlorophyll fluorescence quenching and light allocation in soybean leaves: The cause for midday depression in $\mathrm{CO}_{2}$ assimilation. Sci. Hortic. 2006, 110, 214-218. [CrossRef]

57. Tucci, M.L.S.; Erismann, N.M.; Machado, E.C.; Ribeiro, R.V. Diurnal and seasonal variation in photosynthesis of peach palms grown under subtropical conditions. Photosynthetica 2010, 48, 421-429. [CrossRef]

58. Maxwell, K.; Johnson, G.N. Chlorophyll fluorescence: A practical guide. J. Exp. Bot. 2000, 51, 659-668. [CrossRef]

59. Yamori, W. Photosynthetic response to fluctuating environments and photoprotective strategies under abiotic stress. J. Plant Res. 2016, 129, 379-395. [CrossRef] [PubMed]

60. Alric, J.; Johnson, X. Alternative electron transport pathways in photosynthesis: A confluence of regulation. Curr. Opin. Plant Biol. 2017, 37, 78-86. [CrossRef]

61. Björkman, O.; Demmig, B. Photon yield of $\mathrm{O}_{2}$ evolution and chlorophyll fluorescence characteristics at 779 77k among vascular plants of diverse origins. Planta 1987, 170, 489-504. [CrossRef]

62. Baker, N.R. Chlorophyll fluorescence: A probe of photosynthesis in vivo. Annu. Rev. Plant Biol. 2008, 59, 89-113. [CrossRef] [PubMed]

63. Murchie, E.H.; Niyogi, K.K. Manipulation of photoprotection to improve plant photosynthesis. Plant Physiol. 2011, 155, 86-92. [CrossRef] [PubMed]

64. Li, X.P.; Muller-Moule, P.; Gilmore, A.M.; Niyogi, K.K. PsbS-dependent enhancement of feedback de-excitation protects photosystem II from photoinhibition. Proc. Natl. Acad. Sci. USA 2002, 99, 15222-15227. [CrossRef]

65. Hubbart, S.; Smillie, I.R.A.; Heatley, M.; Swarup, R.; Foo, C.C.; Zhao, L.; Murchie, E.H. Enhanced thylakoid photoprotection can increase yield and canopy radiation use efficiency in rice. Commun. Biol. 2018, 1. [CrossRef] [PubMed]

66. Berg, V.S.; El-Sharkawy, M.A.; Hernandez, A.D.P.; Cock, J.H. Leaf orientation and water relations in cassava. In Annual Meeting of the American Society of Plant Physiologists; Louisiana State University: Baton Rouge, LA, USA, 1986; p. 186.

67. Sawatraksa, W.; Banterng, P.; Jogloy, S. Chlorophyll fluorescence and biomass of four cassava genotypes grown under rain-fed upper paddy field conditions in the tropics. J. Agron. Crop Sci. 2018, 204, 554-565. [CrossRef]

(C) 2019 by the authors. Licensee MDPI, Basel, Switzerland. This article is an open access article distributed under the terms and conditions of the Creative Commons Attribution (CC BY) license (http://creativecommons.org/licenses/by/4.0/). 